\title{
Characterization of the Nucleus, Morphology, and Activity of Interstellar Comet 2I/ Borisov by Optical and Near-infrared GROWTH, Apache Point, IRTF, ZTF, and Keck Observations
}

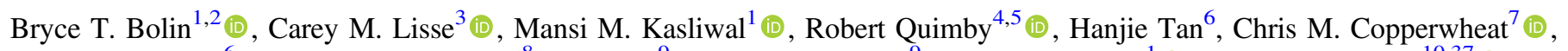
Zhong-Yi Lin ${ }^{6}$, Alessandro Morbidelli ${ }^{8}$, Lyu Abe ${ }^{9}$, Philippe Bendjoya ${ }^{9}$, Kevin B. Burdge ${ }^{1}$ (10), Michael Coughlin ${ }^{10,37}$ (1), Christoffer Fremling ${ }^{1}$ (i), Ryosuke Itoh ${ }^{11}$ (i), Michael Koss ${ }^{12}$ (1), Frank J. Masci ${ }^{2}$ (i), Syota Maeno ${ }^{11}$, Eric E. Mamajek ${ }^{13,14}$ (iD), Federico Marocco ${ }^{2,13}$ (1) , Katsuhiro Murata ${ }^{15}$, Jean-Pierre Rivet ${ }^{9}$, Michael L. Sitko ${ }^{16,17}$ (1), Daniel Stern ${ }^{13}$ (D), David Vernet ${ }^{18}$,

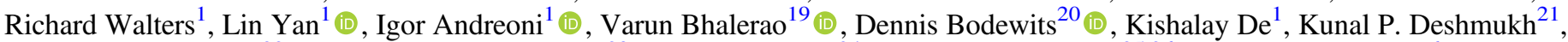

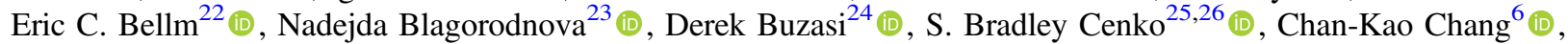
Drew Chojnowski ${ }^{27}$, Richard Dekany ${ }^{28}$, Dmitry A. Duev ${ }^{1}$ (1) , Matthew Graham ${ }^{1}$, Mario Juricie ${ }^{29}$, Shrinivas R. Kulkarni ${ }^{1}$ (D), Thomas Kupfer $^{30}$ (1) , Ashish Mahabal ${ }^{1,31}$, James D. Neill ${ }^{1}$, Chow-Choong Ngeow ${ }^{(1 D}$, Bryan Penprase ${ }^{32}$, Reed Riddle ${ }^{28}$ (D), Hector Rodriguez ${ }^{28}$, Roger M. Smith ${ }^{28}$, Philippe Rosnet ${ }^{33}$, Jesper Sollerman ${ }^{34}$ (i) , and Maayane T. Soumagnac ${ }^{35,36}$ (1)

${ }^{1}$ Division of Physics, Mathematics and Astronomy, California Institute of Technology, Pasadena, CA 91125, USA; bbolin@ caltech.edu

${ }^{2}$ IPAC, Mail Code 100-22, Caltech, 1200 E. California Blvd., Pasadena, CA 91125, USA

3 Johns Hopkins University Applied Physics Laboratory, Laurel, MD 20723, USA

${ }^{4}$ Department of Astronomy, San Diego State University, 5500 Campanile Dr., San Diego, CA 92182, USA

${ }^{5}$ Kavli Institute for the Physics and Mathematics of the Universe (WPI), The University of Tokyo Institutes for Advanced Study, The University of Tokyo, Kashiwa, Chiba 277-8583, Japan

${ }^{6}$ Institute of Astronomy, National Central University, 32001, Taiwan

${ }^{7}$ Astrophysics Research Institute Liverpool John Moores University, 146 Brownlow Hill, Liverpool L3 5RF, UK

${ }^{8}$ Université Côte d'Azur, Observatoire de la Côte d'Azur, CNRS, Laboratoire Lagrange, Boulevard de l'Observatoire, CS 34229, F-06304 Nice cedex 4, France ${ }^{9}$ Université Côte d'Azur, Observatoire de la Côte d'Azur, CNRS, Laboratoire Lagrange, France

${ }^{10}$ LIGO Laboratory, West Bridge, Rm. 257, California Institute of Technology, MC 100-36, Pasadena, CA 91125, USA

${ }^{11}$ Bisei Astronomical Observatory, 1723-70 Ohkur a, Bisei, Ibara, Okayama, 714-1411, Japan

${ }^{12}$ Eureka Scientific, 5248 Valley View Rd., El Sobrante, CA 94803, USA

13 Jet Propulsion Laboratory, California Institute of Technology, 4800 Oak Grove Dr., Pasadena, CA 91109, USA

${ }_{14}$ Department of Physics \& Astronomy, University of Rochester, Rochester, NY 14627, USA

15 Tokyo Institute of Technology, 2 Chome-12-1 Ookayama, Meguro City, Tokyo 152-8550, Japan

${ }^{16}$ Department of Physics, University of Cincinnati, Cincinnati, OH 45221-0011, USA

${ }^{17}$ Space Science Institute, Boulder, CO 80301, USA

${ }^{18}$ Université Côte d'Azur, Observatoire de la Côte d'Azur, UMS Galilée, France

${ }^{19}$ Department of Physics, Indian Institute of Technology Bombay, Powai, Mumbai-400076, India

${ }^{20}$ Physics Department, Leach Science Center, Auburn University, Auburn, AL 36832, USA

${ }^{21}$ Department of Metallurgical Engineering and Materials Science, Indian Institute of Technology Bombay, Powai, Mumbai-400076, India

${ }^{22}$ DIRAC Institute, Department of Astronomy, University of Washington, 3910 15th Ave. NE, Seattle, WA 98195, USA

${ }^{23}$ Department of Astrophysics/IMAPP, Radboud University, Nijmegen, The Netherlands

${ }^{24}$ Department of Chemistry and Physics, Florida Gulf Coast University, 10501 FGCU Blvd. S, Fort Myers, FL 33965, USA

${ }^{25}$ Astrophysics Science Division, NASA Goddard Space Flight Center, 8800 Greenbelt Rd., Greenbelt, MD 20771, USA

${ }^{26}$ Joint Space-Science Institute, University of Maryland, College Park, MD 20742, USA

${ }^{27}$ Department of Astronomy, New Mexico State University, PO Box 30001, MSC 4500, Las Cruces, NM 88001, USA

${ }^{28}$ Caltech Optical Observatories, California Institute of Technology, Pasadena, CA 91125, USA

${ }^{29}$ Department of Astronomy, University of Washington, 3910 15th Ave. NE, Seattle, WA 98195, USA

${ }^{30}$ Kavli Institute for Theoretical Physics, University of California, Santa Barbara, CA 93106, USA

${ }_{32}^{31}$ Center for Data Driven Discovery, California Institute of Technology, Pasadena, CA 91125, USA

${ }^{32}$ Soka University of America, 425 Pauling Hall, 1 University Drive, Aliso Viejo, CA 92656, USA

${ }^{33}$ Université Clermont Auvergne, CNRS/IN2P3, LPC, Clermont-Ferrand, France

${ }^{34}$ Department of Astronomy, Stockholm University, SE-106 91 Stockholm, Sweden

${ }^{35}$ Lawrence Berkeley National Laboratory, 1 Cyclotron Rd., Berkeley, CA 94720, USA

${ }^{36}$ Department of Particle Physics and Astrophysics, Weizmann Institute of Science, Rehovot 76100, Israel

Received 2019 November 5; revised 2020 May 7; accepted 2020 May 12; published 2020 June 17

\begin{abstract}
We present visible and near-infrared (NIR) photometric and spectroscopic observations of interstellar object (ISO) 2I/Borisov taken from 2019 September 10 to 2019 December 20 using the GROWTH, the Apache Point Observatory Astrophysical Research Consortium $3.5 \mathrm{~m}$, and the NASA Infrared Telescope Facility $3.0 \mathrm{~m}$ combined with pre- and postdiscovery observations of 2I obtained by the Zwicky Transient Facility from 2019 March 17 to 2019 May 5. Comparison with imaging of distant solar system comets shows an object very similar to mildly active solar system comets with an outgassing rate of $\sim 10^{27} \mathrm{~mol} \mathrm{~s}^{-1}$. The photometry, taken in filters spanning the visible and NIR range, shows a gradual brightening trend of $\sim 0.03 \mathrm{mag} \mathrm{day}^{-1}$ since 2019 September 10 UTC for a reddish object becoming neutral in the NIR. The light curve from recent and prediscovery data reveals a brightness trend suggesting the recent onset of significant $\mathrm{H}_{2} \mathrm{O}$ sublimation with the comet being active with super volatiles such as $\mathrm{CO}$ at heliocentric distances $>6$ au consistent with its extended morphology. Using the
\end{abstract}

\footnotetext{
${ }^{37}$ David and Ellen Lee Prize Postdoctoral Fellow.
} 
advanced capability to significantly reduce the scattered light from the coma enabled by high-resolution NIR images from Keck adaptive optics taken on 2019 October 4, we estimate a diameter for 2I's nucleus of $\lesssim 1.4 \mathrm{~km}$. We use the size estimates of 1I/'Oumuamua and 2I/Borisov to roughly estimate the slope of the ISO size distribution, resulting in a slope of $\sim 3.4 \pm 1.2$, similar to solar system comets and bodies produced from collisional equilibrium.

Unified Astronomy Thesaurus concepts: Comets (280); Minor planets (1065)

\section{Introduction}

The study of interstellar objects (ISOs) is presently the best opportunity to directly observe the contents of extrasolar circumstellar disks at larger than centimeter-sized scales. Present-day observations are limited to observing the micronsized (e.g., Lisse et al. 2012, 2017) to millimeter-sized (MacGregor et al. 2019) dust contents of extrasolar disks. Indirect observations of macroscopic objects and their volatile contents in debris disks can be obtained through the massive amounts of dust produced by their collision with each other (Meng et al. 2014; Su et al. 2019), their presence around young stars (Chen et al. 2006), or sometimes by their transit of stars (Rappaport et al. 2018), but observing and obtaining the physical properties and volatile contents of specific bodies from other stars has remained elusive.

The second example of a macroscopic body with a definitive interstellar origin to be discovered is 2I/Borisov (2I). Discovered on 2019 August 30 by amateur astronomer Gennadiy Borisov, the hyperbolic orbit with $e \simeq 3.35$ was confirmed on 2019 September 11 (Williams 2019a). Unlike the first ISO to be discovered, 1I/'Oumuamua (Williams 2017), which did not have a cometary appearance in ground-based (Jewitt et al. 2017b; Bolin et al. 2018) or space-based images (Micheli et al. 2018), 2I has a distinct comet-like appearance with a diffuse coma (Jewitt \& Luu 2019). This provides an opportunity to characterize the properties of a cometary interstellar body for the first time.

Initial spectroscopic observations have revealed the presence of $\mathrm{CN}$ and $\mathrm{C}_{2}$ gas in the coma of 2I with gas production rates comparable to solar system comets at similar heliocentric distances, $r_{h}$ (Fitzsimmons et al. 2019; Kareta et al. 2020; Opitom et al. 2019). Using solar system comets as a guide, the production rate of $\mathrm{CN}$ observed in 2I implies a nuclear diameter of $\sim 6 \mathrm{~km}$. The measured size combined with canonical models describing the brightness of $2 \mathrm{I}$ driven by $\mathrm{H}_{2} \mathrm{O}$ or $\mathrm{CO}$ sublimation produces very different results versus heliocentric distance, as a body dominated by $\mathrm{CO}$ sublimation will be active much farther away from the Sun due to CO's much lower enthalpy of sublimation (Meech \& Svoren 2004; Fitzsimmons et al. 2019). Therefore, it may be possible to distinguish between different compositional models of 2I by measuring its brightness at different heliocentric distances covering a wide span of times (e.g., Jewitt et al. 2017a; Meech 2017). This indeed appears to be the case, with Zwicky Transient Facility (ZTF) precovery observations of 2I strongly favoring the activity of the comet being driven by more volatile species than $\mathrm{H}_{2} \mathrm{O}$, such as $\mathrm{CO}$ or $\mathrm{CO}_{2}$ (Ye et al. 2020). In this paper, we build upon these ZTF results and present visible and nearinfrared (NIR) observations of 2I and its morphology; the null result for variability on short-term timescales; estimates of the comet's size, af $\rho$, and dust mass-loss rate; strengthened evidence for activity driven by $\mathrm{CO}$ and $\mathrm{H}_{2} \mathrm{O}$; and an estimate of the ISO cumulative size distribution slope.

\section{Observations}

Since before the official announcement of the hyperbolic orbit of 2I, optical observations were being taken to characterize the object's brightness and refine its orbit. We used the rapidresponse capability of the Global Relay of Observatories Watching Transients Happen (GROWTH) network to organize and schedule observations of 2I. Observations were done at different observatories around the world, all conducted at high airmass, $\gtrsim 2$, just before or during astronomical twilight owing to the small, $43^{\circ}$ solar elongation of the comet in 2019 midSeptember. In addition to the difficulty of observing near twilight and at high airmass, the comet had a fast sky motion of $\sim 1^{\prime \prime}$ minute ${ }^{-1}$, necessitating the use of nonsidereal tracking for the majority of the observations.

We present here the observations of a monitoring campaign led by the GROWTH collaboration (Kasliwal et al. 2019) combined with data from the Apache Point Observatory (APO) Astrophysical Research Consortium (ARC) $3.5 \mathrm{~m}$ telescope, the NASA Infrared Telescope Facility (IRTF) $3.0 \mathrm{~m}$ telescope, ZTF, and Keck Observatory. The time span of our observations is between 2019 March 17 and 2019 December 20 UTC.

\subsection{SED Machine}

The first observations of 2I used in this study were made with the Spectral Energy Distribution Machine (SEDM), operating on the P60 telescope on Palomar (Blagorodnova et al. 2018; Rigault et al. 2019). The SEDM possesses a multiband CCD camera that we used to obtain Sloan Digital Sky Survey (SDSS) $r$-band images in $60 \mathrm{~s}$ exposures of 2I on 2019 September 10 and 11 UTC. The telescope was tracked nonsidereally according to the sky motion of $2 \mathrm{I}$, resulting in background stars that were trailed $\sim 2^{\prime \prime}$. The astrometric positions of 2I were computed and submitted to the MPC to refine the object's orbit (Williams 2019b). The airmass at the time of the observations was $\sim 2$, and the seeing was $\sim 1$ ". 4 in the images taken for the object. This facility is a member of the GROWTH collaboration.

\subsection{APO ARC $3.5 \mathrm{~m}$}

Immediately following the MPC's announcement of the discovery of 2I, we obtained director's discretionary time to observe 2I with the APO ARC $3.5 \mathrm{~m}$. The first observations with the ARC $3.5 \mathrm{~m}$ were made on 2019 September 12 UTC in photometric conditions with the ARCTIC large-format optical CCD camera (Huehnerhoff et al. 2016). The camera was used in full-frame, quad-amplifier readout, $2 \times 2$ binning mode, resulting in a pixel scale of 0 ". 228 . Exposures were each $120 \mathrm{~s}$ long; made in a rotating order of four filters, SDSS griz, in order to mitigate the potential effects of rotational variability on the color calculations (e.g., Hanuš et al. 2018); and dithered by $20^{\prime \prime}$ between exposures of the same filter. In total, five $g$, eight $r$, one $i$, and two $z$ exposures were obtained. The telescope was tracked at the sky motion rate of the comet, resulting in stars 
that were trailed by $\sim 2^{\prime \prime}$. Additional observations were made on 2019 September 27 UTC using the Aspen Apogee Camera in the $R$ band and 2019 October 12 UTC using the ARCTIC camera with Bgriz filters. Seeing was exceptionally good, $\sim 0$ ". 55 in the images taken for the object, on the night of 2019 September 12 UTC; however, the observations were conducted at high airmass and into astronomical twilight, reducing the sensitivity of the observations.

The ARC $3.5 \mathrm{~m}$ was also used to obtain SDSS/Maunakea $z J H K$ photometry of 2I on 2019 September 19 and 27 UTC with the NIC-FPS NIR camera (Vincent et al. 2003). A revolving $Z J H K$ filter sequence was used with a five-point dither pattern. To avoid the effects of the high sky background in the NIR, 40 and $20 \mathrm{~s}$ exposures were used for the $H$ and $K$ filter images, respectively, and 120 and $60 \mathrm{~s}$ exposures were used for the $z$ and $J$ filter images. Up to eight Fowler samples were used per readout to limit readout noise. Seeing was $\sim 1^{\prime \prime}$ or better during the nights of 2019 September 12, 19, and 27 and October 12 and 21 UTC.

\section{3. $L O T$}

Also soon after the discovery of 2I, imaging data were obtained on 2019 September 12 UTC with the $1 \mathrm{~m}$ Lulin Optical Telescope (LOT) using the $2 \mathrm{~K} \times 2 \mathrm{~K}$ SOPHIA camera (Kinoshita et al. 2005) at Lulin Observatory. Data were taken in Johnson-Cousins $V, B, R$, and $I$ bands, and the telescope was tracked nonsidereally at the comet's sky motion rate. The seeing during the observations was $\sim 3$ ". 5 in the images taken for the object, and the airmass was $\sim 2.36$.

\subsection{Bisei Observatory $101 \mathrm{~cm}$}

Images of 2I were obtained at Bisei Observatory ${ }^{38}$ on 2019 September 15 UTC using the $101 \mathrm{~cm}$ reflecting telescope. Images with $60 \mathrm{~s}$ exposure in the Johnson-Cousins $R$ band were obtained using the Astrocam optical camera, and the telescope was tracked at a sidereal rate. Seeing at the time of observations was typically $\sim 2^{\prime \prime}$ in the images taken for the object, and the airmass was $\sim 2$. This facility is a member of the GROWTH collaboration.

\subsection{Liverpool Telescope}

On eight separate nights between 2019 September 18 and 2019 October 15 UTC, observations of 2I were obtained with the $2 \mathrm{~m}$ Liverpool Telescope located at the Observatorio del Roque de los Muchachos. Images were obtained using the IO: $\mathrm{O}$ wide-field camera with a $2 \times 2$ binning and the SDSS $g$ and $r$ filters (Steele et al. 2004). A $30 \mathrm{~s}$ exposure time was used with the telescope tracking the target in a nonsidereal mode. Debiasing and flat-fielding of the data were performed using the automated IO:O pipeline software. Seeing was typically $\sim 1^{\prime \prime}$ in the images taken for the object during the observations, and the airmass was $\sim 1.8-2.0$. This facility is a member of the GROWTH collaboration.

\subsection{Mount Laguna Observatory 40 inch Telescope}

Optical images were obtained with the $1.0 \mathrm{~m}$ telescope at the Mount Laguna Observatory (Smith \& Nelson 1969) on 2019 September 19 and 30 and October 4, 8, 12, and 17 UTC. The

\footnotetext{
${ }^{38}$ http://www.bao.city.ibara.okayama.jp/eng/sisetu.htm
}

E2V 42-40 CCD camera was used to obtain typically six $90 \mathrm{~s}$ exposures in each of the Johnson-Cousins $V$ and $R$ filters each night. Both sidereal and nonsidereal tracking was used, and these produced similar results due to the shortness of the exposures. The seeing during observations was typically $\lesssim 3^{\prime \prime}$, as measured using stars in the images, and the airmass was 1.5-2.0. This facility is a member of the GROWTH collaboration.

\subsection{NASA/IRTF}

On 2019 September 20, 22, and 29 and October 2 UTC, observations of $2 \mathrm{I}$ were obtained with the $3 \mathrm{~m}$ NASA/IRTF located at Maunakea, Hawaii. Images in the $H$ filter and NIR spectra were obtained with a wide 0 ". 8 slit and the lowresolution prism mode of the SpeX prism instrument (Rayner et al. 2003), and $r$-band imagery was also obtained with the MORIS guider camera. The telescope was tracked at comet rate, and long $120 \mathrm{~s}$ exposures were used. The slit was rotated to the optimal azimuth angle in order to reduce differential atmospheric refraction, which would affect the shortest wavelengths in the spectrum the most, when taking prism data for 2I and the standard star. Because of the comet's brief time above the horizon, only four ABBA pairs were obtained on a typically good weather night; the evening of 2019 September 22 UTC was clouded out and little useful data obtained. The most useful data came from the observations on 2019 September 29 UTC, which are described below. The airmass during the time of observation was $\sim 1.5-2.0$. The seeing was $\sim 0$. 8 measured at zenith and worse by $\sim 30 \%$ at the airmass of our observations.

\subsection{ZTF}

The ZTF is a wide-field all-sky survey using Palomar Observatory's P48 Oschin Schmidt telescope (Bellm et al. $2019 b$ ). The mission of the ZTF survey is to discover transients, which include asteroids and comets (Graham et al. 2019). The ZTF camera has a $47 \mathrm{deg}^{2}$ field of view and can reach $r \sim 20.5$ to a signal-to-noise ratio $(\mathrm{S} / \mathrm{N})=5$ depth in a $30 \mathrm{~s}$ exposure, enabling the survey to cover $3800 \mathrm{deg}^{2} \mathrm{hr}^{-1}$. In addition to the GROWTH and APO data, we use prediscovery observations found in the ZTF database to extend the time range of our observations (Masci et al. 2019). Seeing was typically $\sim 2^{\prime \prime}$, and the airmass was $\sim 2$.

Using the latest orbital solution for 2I that was available on 2019 October 2 UTC (Williams 2019c), we used the ZTF database search tool (Masci et al. 2019) to locate images that had overlapping coverage with the trajectory of 2I. The positional uncertainty of 2I in images as far back as 2019 March and May was less than 10"-30". With such a small search area, it became viable to visually spot the detections of the comet in the images where automated software would have missed these detections, i.e., for being too faint, $\mathrm{S} / \mathrm{N} \simeq 2-3$. Therefore, we searched for the detections of 2I by eye in each set of images between 2019 March 17 and 2019 May 5 UTC using the nominal position from JPL HORIZONS as a starting point. The individual detections were very weak, of the order of $\mathrm{S} / \mathrm{N} 2-3$, and in a high sky background owing to the fact that some of them came from observations taken during astronomical twilight.

We identified the prediscoveries on the dates 2019 March 17 and 18 and May 2 and 5 UTC during the public and partnership 
surveys (Bellm et al. 2019a). We used images that were taken with a $30 \mathrm{~s} r$-filter exposure for the prediscovery images. We used two exposures taken on 2019 March 17 UTC, two exposures taken on 2019 March 18 UTC, six exposures taken on 2019 May 2 UTC, and four exposures taken on 2019 May 5 UTC (Ye et al. 2020). In addition to the prediscovery detections, we identified additional postdiscovery detections of 2I in ZTF survey data between 2019 September 11 and 2019 December 20 UTC that were taken in SDSS-like $g$ and $r$ filters (Graham et al. 2019). Seeing was typically $\sim 2^{\prime \prime}$ in the images taken for the object, and the airmass ranged between 1.2 and 2 in the prediscovery images.

\subsection{Keck I Telescope}

We obtained high-resolution images of 2I on 2019 October 4 UTC with the Keck I instrument OSIRIS in imaging mode using laser guide star adaptive optics (AO; Larkin et al. 2006), the first time this instrument and telescope combination had been used to track and observe a comet. The comet was at a heliocentric distance of $2.48 \mathrm{au}$, a topocentric distance of $2.96 \mathrm{au}$, and a phase angle of 18.65 during our observations. Four $60 \mathrm{~s}$ exposures were made in the $K_{p}$ band using the laser guide system with an $r \sim 15$ mag star within $60^{\prime \prime}$ of the comet during the observations. Because of Keck I's $33^{\circ}$ elevation constraint in the azimuth range of 2I, observations had to wait for astronomical twilight to begin. The $K_{p}$ filter $^{39}$ is an NIR filter similar to the Two Micron All Sky Survey (2MASS) $K_{s}$ filter with a central wavelength of $2114.45 \mathrm{~nm}$ and an FWHM bandpass of $307.03 \mathrm{~nm}$. A nearby $r \sim 15$ star was used with the laser guidance system for AO correction while tracking at the sky motion rate of 2I; however, the high airmass of the observations and performance of the laser system resulted in lower image quality than usual. The point-spread function (PSF) FWHM of background stars in OSIRIS images is 0 ". 22-0." 26 measuring in the perpendicular direction of the direction of motion of $2 \mathrm{I}$. The airmass during the time of the observations was $\sim 1.6$.

\subsection{C2PU Facility 1.04 m Omicron Telescope}

Observations of 2I were obtained with the Observatoire de la Côte d'Azur's C2PU $1.04 \mathrm{~m}$ telescope located at Calern on 2019 November 29 UTC. Images were obtained using the $4096 \times 4096$ SBIG STX-16803 CCD camera with a 0". 6 pixel scale in the $R$ bands. A $30 \mathrm{~s}$ exposure time was used with the telescope tracking the target in nonsidereal mode, and the airmass of the observation was $\sim 2.4$. The atmospheric seeing was $\sim 1$ !" 88 in the images taken for the object. Debiasing and flat-fielding was performed on the data using automated software.

\section{Results}

\subsection{Optical Photometry and Colors}

Data collected with the GROWTH and ARC $3.5 \mathrm{~m}$ telescopes were processed using flattened and dark-subtracted images produced by basic methods. Photometric measurements were obtained by using a circular aperture with a projected radius of $10,000 \mathrm{~km}$ at the topocentric distance of the comet, typically $\sim 5^{\prime \prime}$. The typical seeing at our observing locations

\footnotetext{
39 https://www2.keck.hawaii.edu/inst/osiris/technical/filters/filter_ index.html
}

was well under $\sim 5^{\prime \prime}$, measured in the images as described in Table 1. The brightness of the comet was calibrated using the PanSTARRS catalog (Tonry et al. 2012; Flewelling et al. 2016). Johnson-Cousins photometry was calibrated using the PanSTARRS catalog and the filter transformations described in Tonry et al. (2012). We calibrated the photometry with in a frame stars, thus accounting for varying conditions at the high airmass of our observations. Sky subtraction was done using annuli with an inner radius exceeding the extent of the coma by $\gtrsim 10^{\prime \prime}$.

Our team regularly monitored the comet's brightness between 2019 September 10 UTC and 2019 December 20 UTC with telescopes in the GROWTH network at observatories from around the world as described above. The photometric observations cover a span of wavelengths from the $V$ band to $I$ and $z$. To put the photometric measurements on the same scale for comparison, individual Johnson-Cousins filters were converted to the SDSS magnitude system using the colors measured here in griz and VBRI filters (Jordi et al. 2006). The resulting magnitudes are listed in Table 1.

A mosaic of composite images showing the detections of 2I taken by the ARC $3.5 \mathrm{~m}$ on 2019 September 12 UTC in the $g$, $r, i$, and $z$ filters is shown in Figure 1. The comet has a clearly extended appearance with a diffuse tail $\sim 6$ ". 7 long pointing in the $\sim 315^{\circ}$ position angle. Using the stacked images taken by the ARC $3.5 \mathrm{~m}$ on 2019 September 12 UTC, the SDSS griz filter colors of $2 \mathrm{I}$ are $g-r=0.54 \pm 0.06, r-i=0.20 \pm$ 0.04 , and $i-z=-0.23 \pm 0.04$. Immediately after the ARC $3.5 \mathrm{~m}$ observations on 2019 September 12 UTC, BVRI observations were obtained with the LOT, resulting in the following colors: $B-V=0.76 \pm 0.12, \quad V-R=0.55 \pm$ 0.09 , and $R-I=0.37 \pm 0.08$. Converting the ARC $3.5 \mathrm{~m}$ griz colors for 2I to $B V R I$ colors using the transformations in Jordi et al. (2006) results in $B-V=0.69 \pm 0.09$, $V-R=0.40 \pm 0.1$, and $R-I=0.41 \pm 0.07$, which are in good agreement with the LOT BVRI colors and the $B V R I$ colors obtained by Fitzsimmons et al. (2019) and Jewitt \& Luu (2019). An additional observing run on the ARC $3.5 \mathrm{~m}$ was conducted on 2019 October 12 UTC, where Bgriz filtered observations were obtained of 2I with similar colors as measured from data obtained with the ARC $3.5 \mathrm{~m}$ on 2019 September 12 UTC: $g-r=0.63 \pm 0.05, r-i=0.20 \pm$ 0.05 , and $i-z=-0.23 \pm 0.02$. In addition, we calculate $B-V$ colors for data taken on 2019 October 12 UTC by converting our $g$ and $r$ measurements to a $V$ magnitude using the filter transformations for converting SDSS to JohnsonCousins magnitudes. This results in $B-V=0.68 \pm 0.04$, similar to the $B-V$ colors obtained by the LOT on 2019 September 12 UTC and by Jewitt \& Luu (2019).

We extend our color analysis redward of the SDSS $i$ and Cousins $I$ filters, centered at 762 and $880 \mathrm{~nm}$, respectively, to $913 \mathrm{~nm}$ with the inclusion of the SDSS $z$ filter. While the visual spectrum reported by de León et al. (2019) and Hui et al. (2020) shows an overall red appearance, our $g-r$ versus $r-z$ colors of 2I show similarity with neutral and bluish solar system bodies, and 2I does not appear to be as red as outer solar system bodies, such as comets and Kuiper Belt objects (KBOs), with the inclusion of the longer-wavelength $z$-filter data, as seen in Figure 2. This is in contrast with the apparently slightly red color of $2 \mathrm{I}$ in $B-V$ versus $V-R$ color space, as seen in Figure 5 of Jewitt \& Luu (2019), which only goes as red as $635 \mathrm{~nm}$ for the $R$ versus $913 \mathrm{~nm}$ for the $z$-filter $g-r$ versus 
Table 1

Summary of Comet 2I Photometry

\begin{tabular}{|c|c|c|c|c|c|c|c|c|c|c|}
\hline $\begin{array}{l}\text { Date }^{1} \\
\text { UTC } \\
\text { (1) }\end{array}$ & $\begin{array}{c}\text { Telescope }^{2} \\
\text { (2) }\end{array}$ & $\begin{array}{c}r_{h}^{3} \\
(\mathrm{au}) \\
(3)\end{array}$ & $\begin{array}{c}\Delta^{4} \\
(\mathrm{au}) \\
(4)\end{array}$ & $\begin{array}{c}\alpha^{5} \\
(\mathrm{deg}) \\
(5)\end{array}$ & $\begin{array}{c}\nu^{6} \\
(\operatorname{deg}) \\
(6)\end{array}$ & $\begin{array}{c}\text { Filter }^{7} \\
\text { (7) }\end{array}$ & $\begin{array}{l}\text { Mag. } \\
\text { (8) }\end{array}$ & $\begin{array}{c}\sigma_{\text {mag }}^{9} \\
(9)\end{array}$ & $\begin{array}{c}\theta_{s}^{10} \\
(\operatorname{arcsec}) \\
(10)\end{array}$ & $\begin{array}{l}\chi_{\mathrm{am}}^{11} \\
(11)\end{array}$ \\
\hline 2019 Mar 17 & ZTF & 6.0 & 6.1 & 9.3 & 277.6 & $r$ & 20.71 & 0.37 & 2.37 & 1.32 \\
\hline 2019 Mar 18 & ZTF & 6.0 & 6.1 & 9.3 & 277.7 & $r$ & 21.01 & 0.37 & 2.18 & 1.27 \\
\hline 2019 May 2 & ZTF & 5.2 & 5.8 & 8.2 & 281.8 & $r$ & 20.30 & 0.18 & 2.11 & 1.90 \\
\hline 2019 May 5 & ZTF & 5.1 & 5.8 & 8.0 & 282.0 & $r$ & 20.66 & 0.31 & 2.53 & 2.01 \\
\hline 2019 Sep 10 & SEDM & 2.8 & 3.48 & 13.9 & 308.6 & $r$ & 17.91 & 0.05 & 2.11 & 1.89 \\
\hline 2019 Sep 11 & SEDM & 2.8 & 3.45 & 14.1 & 309.2 & $r$ & 17.71 & 0.04 & 1.74 & 1.68 \\
\hline 2019 Sep 11 & ZTF & 2.8 & 3.45 & 14.1 & 309.2 & $g$ & 18.43 & 0.06 & 1.92 & 1.40 \\
\hline 2019 Sep 12 & ARC $3.5 \mathrm{~m}$ & 2.78 & 3.43 & 14.3 & 309.6 & $g$ & 18.29 & 0.04 & 0.66 & 2.34 \\
\hline 2019 Sep 12 & ARC $3.5 \mathrm{~m}$ & 2.78 & 3.43 & 14.3 & 309.6 & $r$ & 17.75 & 0.04 & 0.62 & 2.15 \\
\hline 2019 Sep 12 & ARC $3.5 \mathrm{~m}$ & 2.78 & 3.43 & 14.3 & 309.6 & $i$ & 17.55 & 0.01 & 0.58 & 2.32 \\
\hline 2019 Sep 12 & ARC $3.5 \mathrm{~m}$ & 2.78 & 3.43 & 14.3 & 309.6 & $z$ & 17.78 & 0.03 & 0.56 & 2.21 \\
\hline 2019 Sep 12 & LOT & 2.78 & 3.43 & 14.3 & 309.6 & V & 18.01 & 0.05 & 3.50 & 2.36 \\
\hline 2019 Sep 12 & LOT & 2.78 & 3.43 & 14.3 & 309.6 & B & 18.77 & 0.1 & 3.50 & 2.36 \\
\hline 2019 Sep 12 & LOT & 2.78 & 3.43 & 14.3 & 309.6 & $R$ & 17.47 & 0.04 & 3.50 & 2.36 \\
\hline 2019 Sep 12 & LOT & 2.78 & 3.43 & 14.3 & 309.6 & $I$ & 17.09 & 0.04 & 3.50 & 2.36 \\
\hline 2019 Sep 15 & Bisei & 2.73 & 3.37 & 14.9 & 310.7 & $R$ & 17.41 & 0.06 & 2.05 & 2.11 \\
\hline 2019 Sep 18 & Liverpool & 2.69 & 3.30 & 15.5 & 311.9 & $g$ & 18.23 & 0.17 & 1.03 & 2.25 \\
\hline 2019 Sep 19 & MLO $1.0 \mathrm{~m}$ & 2.68 & 3.27 & 15.7 & 312.3 & $r$ & 17.86 & 0.02 & 3.53 & 2.08 \\
\hline 2019 Sep 21 & ZTF & 2.65 & 3.23 & 16.0 & 313.1 & $r$ & 17.82 & 0.04 & 1.76 & 1.76 \\
\hline 2019 Sep 22 & ZTF & 2.64 & 3.21 & 16.2 & 313.6 & $g$ & 17.72 & 0.06 & 2.53 & 1.72 \\
\hline 2019 Sep 22 & ZTF & 2.64 & 3.21 & 16.2 & 313.6 & $r$ & 18.33 & 0.13 & 2.16 & 2.21 \\
\hline 2019 Sep 27 & ARC $3.5 \mathrm{~m}$ & 2.56 & 3.11 & 17.2 & 315.7 & $R$ & 17.45 & 0.04 & 1.87 & 2.25 \\
\hline 2019 Sep 30 & MLO $1.0 \mathrm{~m}$ & 2.52 & 3.04 & 17.8 & 317.1 & $r$ & 17.51 & 0.02 & 3.49 & 2.32 \\
\hline 2019 Oct 1 & ZTF & 2.51 & 3.02 & 18.0 & 317.5 & $r$ & 17.36 & 0.03 & 2.20 & 2.35 \\
\hline 2019 Oct 2 & ZTF & 2.50 & 2.99 & 18.2 & 318.0 & $g$ & 17.83 & 0.04 & 2.24 & 2.20 \\
\hline 2019 Oct 2 & ZTF & 2.50 & 2.99 & 18.2 & 318.0 & $r$ & 17.25 & 0.04 & 1.94 & 1.80 \\
\hline 2019 Oct 4 & MLO $1.0 \mathrm{~m}$ & 2.47 & 2.95 & 18.64 & 318.9 & V & 17.80 & 0.02 & 3.75 & 1.99 \\
\hline 2019 Oct 4 & MLO $1.0 \mathrm{~m}$ & 2.47 & 2.95 & 18.64 & 318.9 & $r$ & 17.32 & 0.01 & 3.62 & 1.95 \\
\hline 2019 Oct 4 & Liverpool & 2.47 & 2.95 & 18.64 & 318.9 & $g$ & 17.89 & 0.02 & 1.12 & 1.97 \\
\hline 2019 Oct 4 & Liverpool & 2.47 & 2.95 & 18.64 & 318.9 & $r$ & 17.31 & 0.01 & 1.17 & 1.94 \\
\hline 2019 Oct 5 & ZTF & 2.46 & 2.94 & 18.85 & 319.4 & $r$ & 17.19 & 0.04 & 1.97 & 2.02 \\
\hline 2019 Oct 8 & MLO $1.0 \mathrm{~m}$ & 2.42 & 2.87 & 19.45 & 320.8 & V & 17.73 & 0.03 & 2.82 & 2.17 \\
\hline 2019 Oct 8 & MLO $1.0 \mathrm{~m}$ & 2.42 & 2.87 & 19.45 & 320.8 & $r$ & 17.21 & 0.01 & 2.95 & 2.22 \\
\hline 2019 Oct 8 & Liverpool & 2.42 & 2.87 & 19.45 & 320.8 & $g$ & 17.75 & 0.03 & 1.03 & 2.01 \\
\hline 2019 Oct 8 & Liverpool & 2.42 & 2.87 & 19.45 & 320.8 & $r$ & 17.15 & 0.01 & 1.07 & 1.99 \\
\hline 2019 Oct 10 & Liverpool & 2.40 & 2.83 & 19.85 & 321.8 & $g$ & 17.61 & 0.01 & 0.99 & 1.98 \\
\hline 2019 Oct 10 & Liverpool & 2.40 & 2.83 & 19.85 & 321.8 & $r$ & 17.13 & 0.01 & 1.08 & 1.93 \\
\hline 2019 Oct 11 & ZTF & 2.38 & 2.81 & 20.1 & 322.4 & $g$ & 17.71 & 0.04 & 2.99 & 2.03 \\
\hline 2019 Oct 12 & ARC $3.5 \mathrm{~m}$ & 2.37 & 2.79 & 20.25 & 322.9 & B & 18.04 & 0.04 & 0.88 & 1.56 \\
\hline 2019 Oct 12 & ARC $3.5 \mathrm{~m}$ & 2.37 & 2.79 & 20.25 & 322.9 & $g$ & 17.74 & 0.05 & 0.73 & 1.71 \\
\hline 2019 Oct 12 & ARC $3.5 \mathrm{~m}$ & 2.37 & 2.79 & 20.25 & 322.9 & $r$ & 17.11 & 0.05 & 0.78 & 2.02 \\
\hline 2019 Oct 12 & ARC $3.5 \mathrm{~m}$ & 2.37 & 2.79 & 20.25 & 322.9 & $i$ & 16.94 & 0.02 & 0.82 & 2.12 \\
\hline 2019 Oct 12 & ARC $3.5 \mathrm{~m}$ & 2.37 & 2.79 & 20.25 & 322.9 & $z$ & 17.14 & 0.05 & 0.75 & 1.62 \\
\hline 2019 Oct 12 & MLO $1.0 \mathrm{~m}$ & 2.37 & 2.79 & 20.25 & 322.9 & V & 17.55 & 0.04 & 2.78 & 1.74 \\
\hline 2019 Oct 12 & MLO $1.0 \mathrm{~m}$ & 2.37 & 2.79 & 20.25 & 322.9 & $r$ & 17.27 & 0.04 & 2.56 & 1.83 \\
\hline 2019 Oct 14 & Liverpool & 2.35 & 2.76 & 20.66 & 323.9 & $r$ & 17.14 & 0.04 & 1.10 & 1.9 \\
\hline 2019 Oct 14 & ZTF & 2.35 & 2.76 & 20.66 & 323.9 & $g$ & 17.74 & 0.08 & 2.00 & 1.37 \\
\hline 2019 Oct 14 & ZTF & 2.35 & 2.76 & 20.66 & 323.9 & $r$ & 17.16 & 0.04 & 2.01 & 1.77 \\
\hline 2019 Oct 15 & ZTF & 2.34 & 2.74 & 20.86 & 324.4 & $r$ & 17.18 & 0.05 & 3.84 & 1.79 \\
\hline 2019 Oct 17 & MLO $1.0 \mathrm{~m}$ & 2.32 & 2.70 & 21.26 & 325.5 & $V$ & 17.46 & 0.04 & 2.93 & 1.68 \\
\hline 2019 Oct 17 & MLO $1.0 \mathrm{~m}$ & 2.32 & 2.70 & 21.26 & 325.5 & $r$ & 17.19 & 0.04 & 3.12 & 1.76 \\
\hline 2019 Oct 21 & ARC $3.5 \mathrm{~m}$ & 2.28 & 2.62 & 22.04 & 327.7 & $r$ & 16.99 & 0.02 & 2.21 & 2.13 \\
\hline 2019 Oct 29 & ZTF & 2.20 & 2.48 & 23.56 & 332.4 & $r$ & 16.84 & 0.01 & 1.92 & 1.59 \\
\hline 2019 Nov 3 & ARC $3.5 \mathrm{~m}$ & 2.16 & 2.40 & 24.46 & 335.5 & $r$ & 16.76 & 0.02 & 0.96 & 2.58 \\
\hline 2019 Nov 5 & ZTF & 2.14 & 2.37 & 24.79 & 336.7 & $r$ & 16.61 & 0.03 & 1.74 & 1.68 \\
\hline 2019 Nov 8 & ZTF & 2.12 & 2.32 & 25.29 & 338.7 & $r$ & 16.76 & 0.03 & 1.92 & 1.40 \\
\hline 2019 Nov 12 & ZTF & 2.09 & 2.26 & 25.91 & 341.3 & $r$ & 16.76 & 0.04 & 2.13 & 2.20 \\
\hline 2019 Nov 17 & ZTF & 2.06 & 2.20 & 26.62 & 344.7 & $r$ & 16.88 & 0.04 & 2.67 & 1.40 \\
\hline 2019 Nov 27 & ZTF & 2.02 & 2.08 & 27.76 & 351.7 & $g$ & 17.29 & 0.0 & 4.18 & 1.65 \\
\hline 2019 Nov 29 & $\mathrm{C} 2 \mathrm{PU}$ & 2.01 & 1.99 & 27.93 & 353.1 & $r$ & 16.88 & 0.03 & 1.88 & 2.40 \\
\hline 2019 Dec 20 & $\mathrm{ZTF}$ & 2.03 & 1.94 & 28.63 & 8.2 & $g$ & 17.50 & 0.06 & 2.51 & 1.90 \\
\hline
\end{tabular}

Note. Columns: (1) observation date, (2) observatory, (3) heliocentric distance, (4) topocentric distance, (5) phase angle, (6) true anomaly, (7) filter, (8) $10^{4} \mathrm{~km}$ aperture mag, (9) $1 \sigma$ mag uncertainty, (10) in-image seeing of observations, (11) airmass of observations. 
$r-z$ color space. We must caution that the comparison of the colors between 2I and known solar system comets can be affected by the fact that comet dust for active comets can modify their apparent color compared to inactive bodies ( $\mathrm{Li}$ et al. 2013). We also further caution that although 2I appears neutral to reddish with the addition of longer wavelengths in the $g-r$ versus $r-z$ color space compared to $B-V$ versus $V-R$ color space, the interpretation of the colors of small bodies is limited by the fact that many solar system bodies appear neutral in optical colors spanning wavelengths of 477-913 nm for filters $g$ to $z$ (Bus \& Binzel 2002). However, solar system objects that appear to be neutral in optical wavelengths can be revealed to be much redder with the inclusion of even longer wavelength data in the NIR range (e.g., DeMeo et al. 2009; Schwamb et al. 2019), as further discussed in Section 3.2. We wish to reiterate that this comparison of colors with inactive bodies is for reference only. In addition, 2I exhibits colors that are markedly different from active solar system objects, being less red in $r-z$ color, which may indicate that the color of the dust of $2 \mathrm{I}$ is different from solar system comets.

We increased the range of our long-term light curve by using prediscovery observations of 2I found in $r$-filter images from the ZTF survey spanning 2019 March to May. Ye et al. (2020) presented these measurements and analysis; here we repeat their extraction from raw data both as a cross-check and for methodological consistency reasons.

The prediscovery detections from ZTF were stacked in the individual images, increasing their $\mathrm{S} / \mathrm{N}$ by locating detections in several overlapping images taken on the same night that were processed to remove static sources. The limiting magnitude in the image stacks was $r \sim 21.5$ for the $60 \mathrm{~s}$ equivalent exposure time image stacks taken in 2019 March and $r \sim 22.5$ and 22 for the 180 and 120 s equivalent exposure time image stacks taken on 2019 May 2 and 5, respectively. The image stacks showing the individual detections taken on 2019 March 17 UTC and 2019 May 2 UTC are shown in the bottom panels of Figure 1. In addition to the photometry, the prediscovery detections were measured astrometrically and submitted to the MPC, allowing for the orbital arc to be significantly extended by several months, improving its accuracy, and for use by the community to study 2I. The photometry from the postdiscovery observations by the GROWTH and ZTF telescopes is listed in Table 1.

\subsection{NIR Photometry and Spectrum}

From our VisNIR observations with the ARC 3.5, cometary morphology is evident in the $z$ image, but the cometary appearance is suppressed in the longer-wavelength $J H K$ images as seen in Figure 3 due to light scattering by cometary dust being less efficient at longer wavelengths (Fernández et al. 2013; Bauer et al. 2017). The $z$ and $J H K$ photometry were calibrated using the PanSTARRS (Chambers et al. 2016) and 2MASS (Skrutskie et al. 2006) catalogs. We measure magnitudes $z=17.57 \pm 0.05, \quad J=16.80 \pm 0.05$, $H=16.01 \pm 0.09$, and $K=15.81 \pm 0.10$. Combined with the $R$-filter observation also taken on 2019 September 27 UTC, the resulting colors are, after converting the $R$ measurement to $r=17.60 \pm 0.04, r-z=0.03 \pm 0.06, r-J=0.80 \pm 0.06$, $z-J=0.77 \pm 0.07, \quad J-H=0.79 \pm 0.10, \quad$ and $H-K=$ $0.20 \pm 0.13$, similar to neutral solar system objects and distinct from very red outer solar system objects (Schwamb et al. 2019).
As seen in Figure 3 from Bannister et al. (2017), the rough dividing line in $r-J$ separating outer solar system objects from inner solar system objects is $r-J \gtrsim 1.2$, where the $r-J$ of $2 \mathrm{I}$ is $\sim 0.8$.

We made a pair-subtracted stack of the four $120 \mathrm{~s}$ ABBA sequence SpeX prism exposures of $2 \mathrm{I}$, resulting in the composite spectrum seen in the top panel of Figure 3. The full compliment of gri and $z J H K$ photometry from 2019 October 12 and 2019 September 27 UTC are overplotted on top of the NIR spectrum, showing agreement with the visible portion with the visible spectrum of de León et al. (2019). The spectrum was adjusted to the photometric points. The IR color of $2 \mathrm{I}$, as determined by the continuum slope of the prism spectra, was found to be neutral gray, in agreement with the $r z J H K$ colors, in contrast with Yang et al. (2019). No definitive absorption or emission lines were found within the errors of the measurements, similar to the lack of emission lines seen in the spectra of solar system comets and asteroids in the 0.7-2.5 $\mu \mathrm{m}$ range (Feldman et al. 2004).

The colors are typical of optically reddish objects containing refractory organics and silicates that become NIR-neutral because of the presence of water ice (Yang et al. 2009; Snodgrass et al. 2017). Because the flux of 2I is dominated by its coma, as discussed in Section 3.1, we can infer, by analogy with solar system comet spectra, that the 2I NIR spectrum coma dust contains silicates, refractory organics, and water ice (Protopapa et al. 2014; Bockelée-Morvan et al. 2017), though recent observations suggest that the main driver of the activity is $\mathrm{CO}$ and is likely responsible for driving the dust production (Bodewits et al. 2020).

From our OSIRIS observations with Keck I, a composite stack of the $K_{p}$ images of 2I is shown in Figure 4. No extended coma or tail features are evident in the OSIRIS images taken on 2019 October 4 UTC owing to the low surface brightness of these features in $K$-band wavelengths similar to the NIR wavelength images taken by the ARC $3.5 \mathrm{~m}$ on 2019 September 27 UTC, as seen in the bottom panel of Figure 3. We estimate the apparent brightness of $2 \mathrm{I}$ in the AO $K_{p}$ images measured to be $m_{K_{p}}=15.68 \pm 0.06$ using a 4 ." 7 circular aperture with a projected radius of $10,000 \mathrm{~km}$ at the topocentric distance of the comet of 2.96 au on 2019 October 4 and the zero-point of 27.6 determined for the $K_{p}$ of the OSIRIS instrument. ${ }^{40}$

\subsection{Long-term Light Curve and Volatile-driven Activity}

Due to the density and slow crossing time of dust within 2I's coma at the scale of our ground-based observations, as discussed in Jewitt \& Luu (2019), measuring any short-term light-curve variations on the order of hours to tens of hours caused by the rotation of the comet's nucleus is difficult. However, other effects on the comet's brightness can happen on longer time spans of weeks to months, such as outbursts, seasonal effects, or changes in its activity due to the sublimation of different volatile species that become active at different heliocentric distances along the comet's orbit (Hughes 1990; Li et al. 2016; Keller et al. 2017; Womack et al. 2017). Because these effects can take weeks to months, a comet needs to be monitored over a long time period, requiring the dedication of observers to make regular observations of the comet. A detailed discussion of the long-term light curve's implication for the activity of 2I follows.

\footnotetext{
${ }^{40}$ https://www2.keck.hawaii.edu/inst/osiris/OSIRIS_Manual_v2.2.pdf
} 

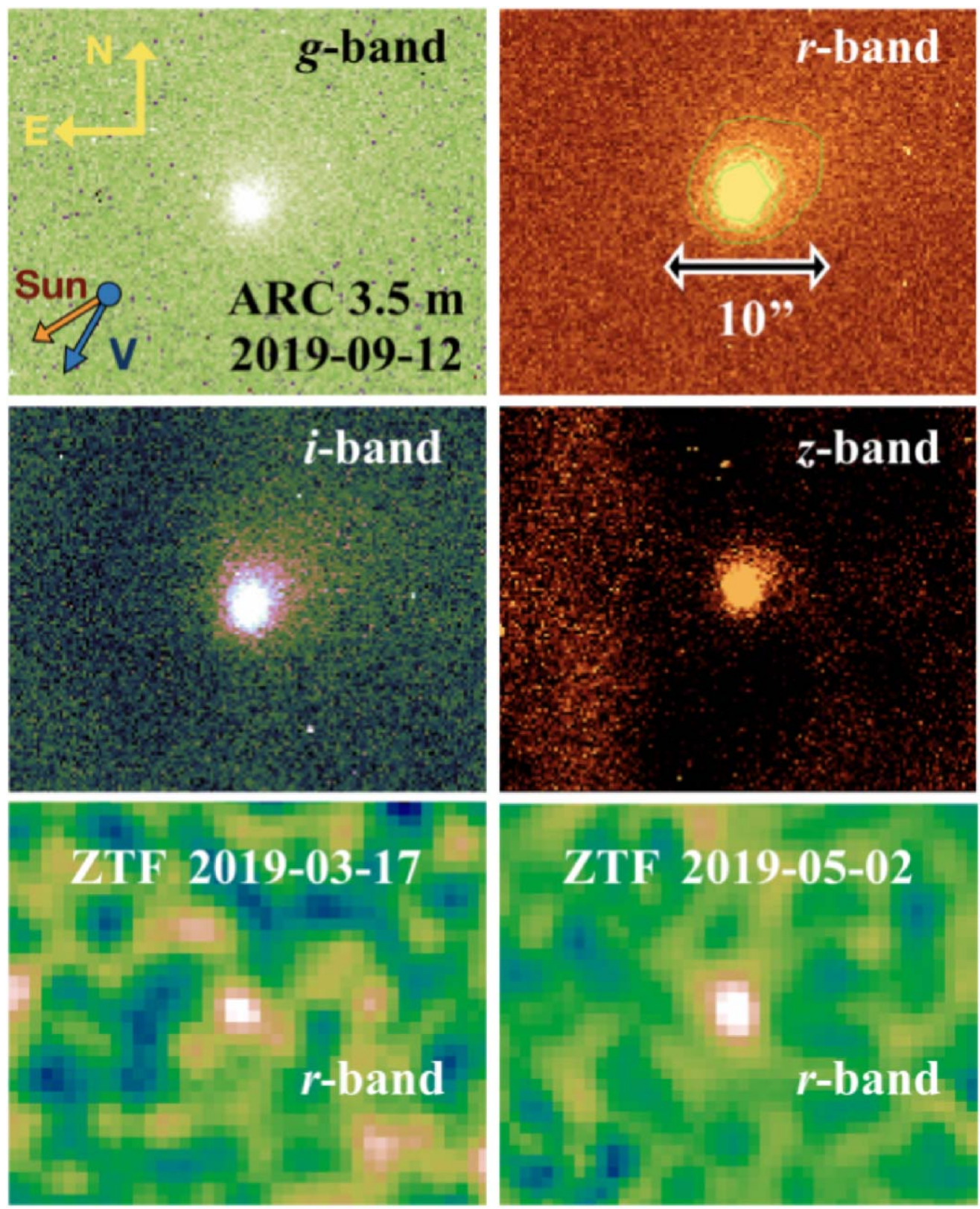

Figure 1. Mosaic of $g, r, i$, and $z$ images of 2I taken with the ARC $3.5 \mathrm{~m}$ on 2019 September 12 UTC and prediscovery ZTF images from 2019 March 17 and 2019 May 2 UTC. Top left panel: composite stack of five $120 \mathrm{~s} g$-filter exposures with the orbital velocity and solar directions. Top right panel: composite stack of eight $120 \mathrm{~s} r$-filter exposures and showing the extent of the comet's tail limited by sky background. Middle left panel: single $120 \mathrm{~s} i$-filter exposure. Middle right panel: stack of two $z$-filter exposures. The nearby background is irregular due to incomplete removal of fringes. Calibration stars were carefully chosen that were not affected by these fringe removal artifacts. Bottom left and right panels: prediscovery detections of 2I from 2019 March 17 and 2019 May 2 UTC. The 2019 March 17 UTC data is a stack of two images with an equivalent exposure time of $60 \mathrm{~s}$. The 2019 May 2 UTC data is a stack of six images with an equivalent exposure time of $180 \mathrm{~s}$. Both of these ZTF image stacks have been spatially smoothed to enhance faint features in the image. The artifact at the bottom of the 2019 March 17 UTC image is a star subtraction artifact. No extended coma or tail features are evident in the prediscovery image stacks owing to the low surface brightness of these features at the time of observation. A green color scale was chosen for the ZTF $r$-filter observations to more clearly highlight these faint detections compared to the surrounding background.

The light curve of equivalent $r$ magnitudes is plotted in Figure 5. As of writing, the brightness of 2I, plotted as orange squares, appears to follow the trend predicted by Fitzsimmons et al. (2019) for an $\mathrm{H}_{2} \mathrm{O}$-dominated comet, plotted as a blue line, best seen in the inset plot zoomed in on $-52^{\circ}$ to $-32^{\circ}$ in Figure 5. The activity for a $\mathrm{CO}_{2}$-dominated comet is plotted as an orange line. Both of these activity models from Fitzsimmons et al. (2019) are based on assuming a nucleus diameter of $\sim 1 \mathrm{~km}$ and activity consistent with solar system comets using the measured $\mathrm{CN}$ activity to estimate the production rate of other volatile species (A'Hearn et al. 1995). In addition, the activity model assumes that $100 \%$ of the comet's surface is active and dust grain properties are similar to solar system comets. There was a recent rise in brightness as $2 \mathrm{I}$ approached the water-ice line at heliocentric distance $r_{h}=2.5$ au on 2019 October 2 UTC that may correspond to the increase in the sublimation rate of $\mathrm{H}_{2} \mathrm{O}$ as the comet approaches the Sun (Meech \& Svoren 2004; Jewitt et al. 2015).

Extrapolating the $\mathrm{H}_{2} \mathrm{O}$ brightness model backward to the prediscovery data taken by ZTF in 2019 March and May, when the comet was at a heliocentric distance of 6.03 and $5.09 \mathrm{au}$, respectively, predicts a much fainter magnitude of $r \sim 26$ than 


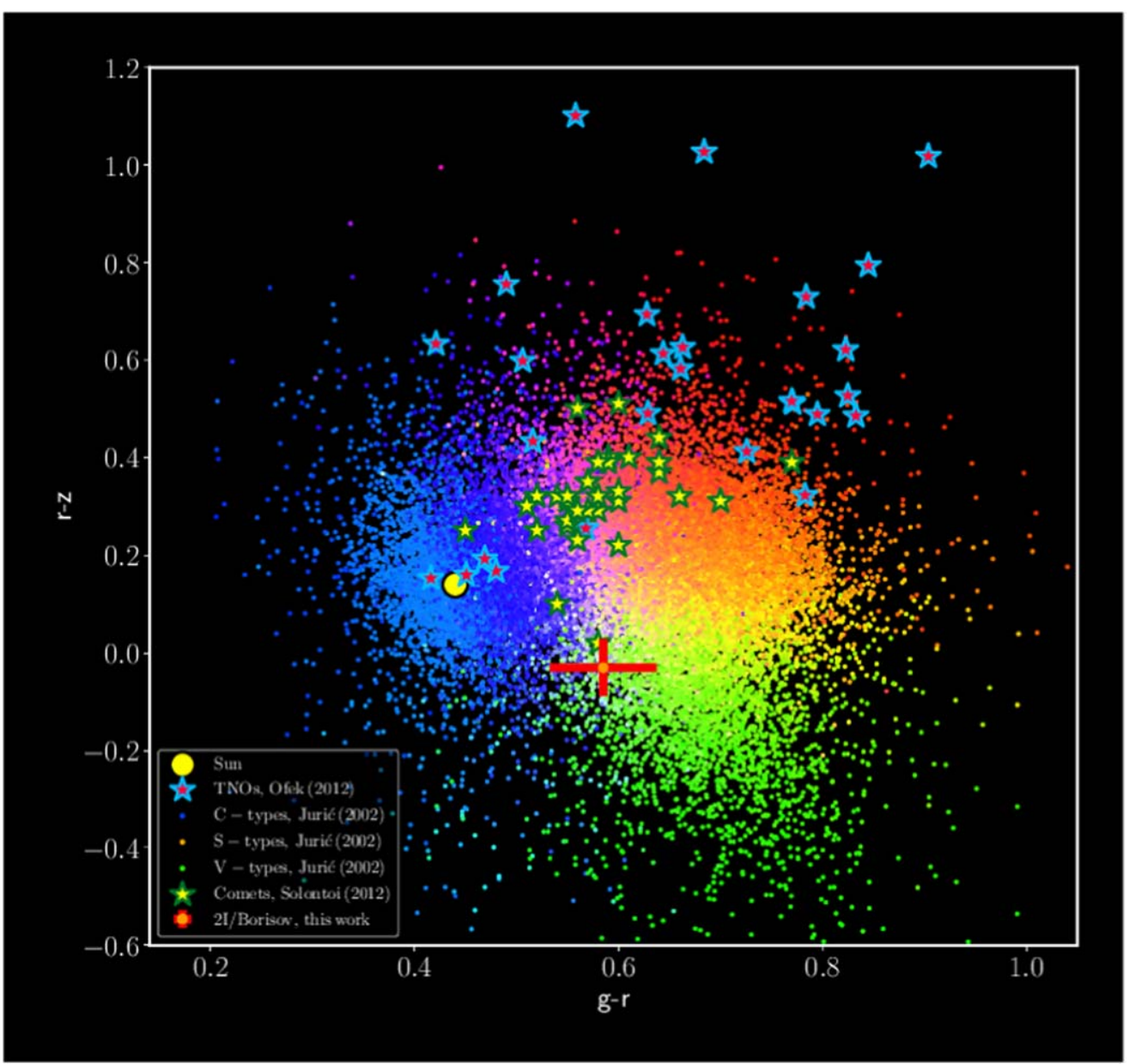

Figure 2. The $g-r$ vs. $r-z$ colors of 2I plotted with the $g-r$ and $r-z$ colors of other solar system bodies, including inner solar system asteroids such as C, S, and V types (Ivezić et al. 2001; Jurić et al. 2002; DeMeo \& Carry 2013); active comets (Solontoi et al. 2012); and KBOs (Ofek 2012). The colorization scheme of data points for asteroids by their griz colors is adapted from Ivezić et al. (2002). We note that the comparison of the colors of 2I to active comets in Solontoi et al. (2012) is the most appropriate comparison, rather than inactive bodies, since the colors of 2I are most representative of its dust, rather than bare nucleus. The colors of inactive bodies are present for comparison only.

the observed magnitude of $r=20.5-21.0$. As shown by Ye et al. (2020) and confirmed by our work, the actual observed prediscovery $r$ magnitudes are much closer to the brightness model predicted for a comet that has its activity dominated by $\mathrm{CO}$ than $\mathrm{H}_{2} \mathrm{O}$ (Fitzsimmons et al. 2019). This is supported by the fact that $\mathrm{H}_{2} \mathrm{O}$ is very weakly sublimating at temperatures $\lesssim 150 \mathrm{~K}$ at a heliocentric distance $>3.5 \mathrm{au}$, while $\mathrm{CO}$ can become volatile much further from the Sun at heliocentric distances exceding 10-100 au (Meech \& Svoren 2004).

However, the prediscovery photometry may also be compatible with $\mathrm{CO}_{2}$-driven activity where $\mathrm{CO}_{2}$ can become active at $>13$ au (Womack et al. 2017; Ye et al. 2020). As discussed in Section 3.4, a significant production rate of $\mathrm{H}_{2} \mathrm{O}$ is inferred from the observed production of $\mathrm{CN}$ and $\mathrm{C}_{2}$ gas (Fitzsimmons et al. 2019; Kareta et al. 2020) and is $\sim 100$ $\mathrm{kg} \mathrm{s}^{-1}$ comparable, though larger than the $\mathrm{H}_{2} \mathrm{O} \sim 20 \mathrm{~kg} \mathrm{~s}^{-1}$ production inferred from the detection of the [O I] $6300 \AA$ line taken at further heliocentric distances (McKay et al. 2020). Since our photometric light curve suggests that the activity of 2I is partially driven by $\mathrm{CO}$, we expect the mass loss of $\mathrm{CO}$ to also be much higher than the mass loss from dust in the $\sim 10-100 \mathrm{~kg} \mathrm{~s}^{-1}$ range as it approaches perihelion. The ratio of $\mathrm{CO}$ to $\mathrm{H}_{2} \mathrm{O}$ has been shown to be $>130 \%$, as revealed by recent Hubble Space Telescope (HST) and Atacama Large
Millimeter/submillimeter Array observations (Bodewits et al. 2020; Cordiner et al. 2020), much higher than the typical $<30 \%$ of solar system comets (Paganini et al. 2014; Meech 2017).

The difference between the observed brightness of 2I in the prediscovery data is even larger for a bare, inactive $\sim 1.4 \mathrm{~km}$ diameter nucleus, as seen from the black dashed-dotted line in Figure 5. In addition, there appears to be an $\sim 0.2$ mag change in brightness in the light curve between 2019 September 20 and 2019 October 3 UTC corresponding to true anomaly angles $-47^{\circ}$ and $-42^{\circ}$, deviating from the trend predicting the brightness for an $\mathrm{H}_{2} \mathrm{O}$-dominated comet as seen in Figure 5, and possibly indicating a change in the activity of the comet. We must caution that the height of the curves is also dependent on the size of the nucleus, and the activity could be compatible with a slight increase in nucleus size and a corresponding decrease in the water production rate.

Concerning the outgassing models used to constrain the activity, it is important to note that our suggestion of initial COdriven outgassing activity transitioning to $\mathrm{H}_{2} \mathrm{O}$-driven activity is not dependent on 2I's nucleus size or fractional active outgassing area. The fractional active outgassing scales the $\mathrm{CO}$ $+\mathrm{H}_{2} \mathrm{O}$ model; once set, this scale is fixed. It is the relative shape of our measured 2I long-term light curve and the upward 

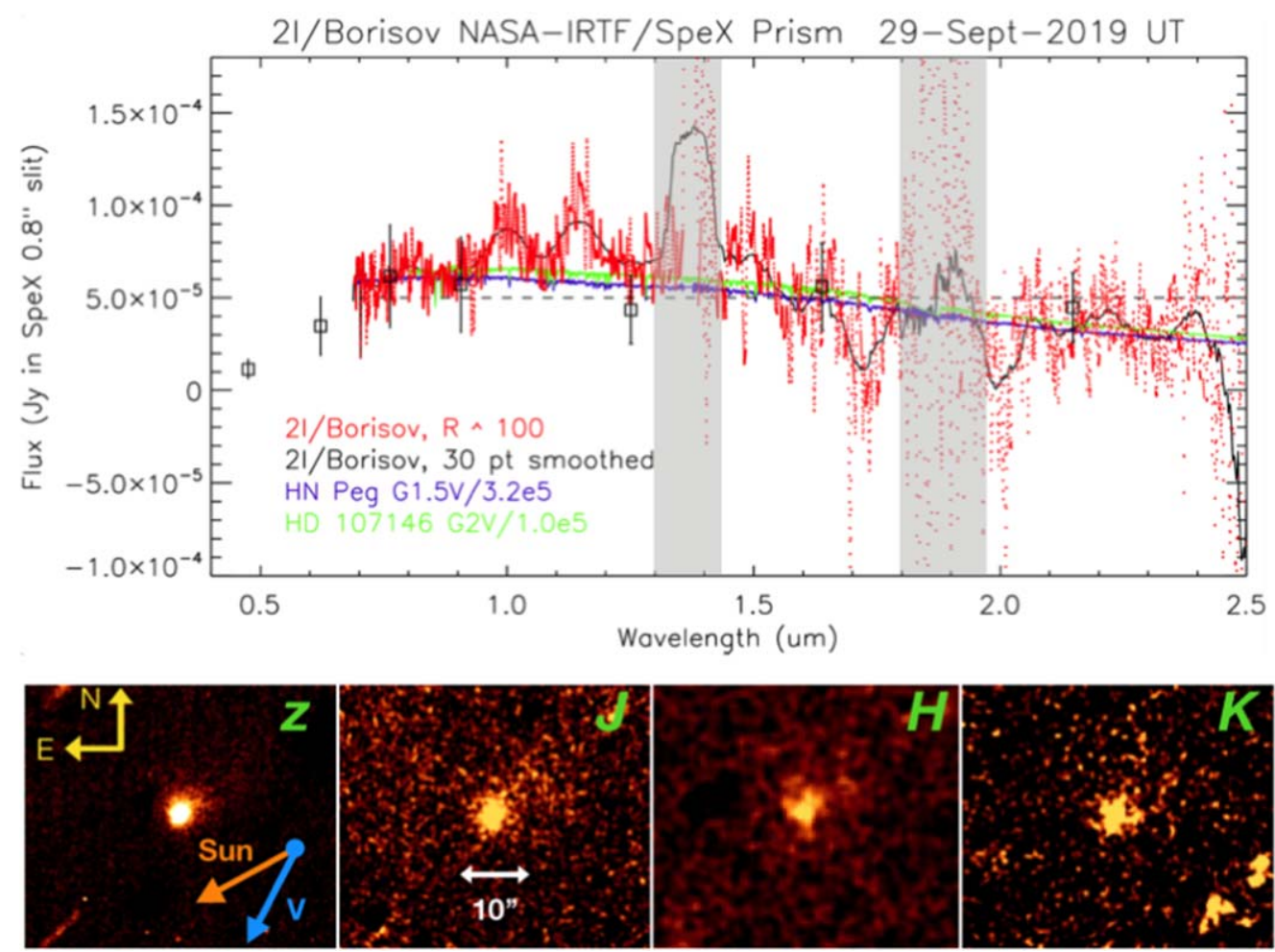

Figure 3. Top panel: scaled flux IRTF SpeX spectrum of 2 I taken on 2019 September 29 UTC. The red line is an $R \sim 100$ spectrum between 0.7 and $2.5 \mu$ m from two ABBA $120 \mathrm{~s}$ pair subtractions. The black line is the smoothed spectrum of 2I with a $30 \mathrm{pt}(\sim 50 \mathrm{~nm})$ running mean. The blue and green lines correspond to G1.5V and G2V analog stars HN Peg and HD 107146. The SED is overall reddish-neutral with some slight deviations in the 0.9-1.2 $\mu \mathrm{m}$ range. The gri fluxes obtained in observations on 2019 October 12 and the $z J H K$ fluxes obtained in observations on 2019 September 27 with the ARC $3.5 \mathrm{~m}$ are overplotted on the spectrum and in rough agreement with the spectrum. Emission features at $\sim 1.4$ and $\sim 1.8 \mu \mathrm{m}$ are of terrestrial atmospheric origin. Bottom panel: $z J H K$ image stacks of 2I taken on 2019 September 27 UTC with NIC-FPS on the ARC $3.5 \mathrm{~m}$. The $z$ and $J$ images are a $600 \mathrm{~s}$ robust mean stack, and the $H$ and $K$ images are $200 \mathrm{~s}$ robust mean stacks. All images have been spatially smoothed to enhance faint features. The north and south directions and the solar and orbital velocity directions are indicated on the $z$ band panel. Regions of the spectrum degraded by sky absorption are grayed out.

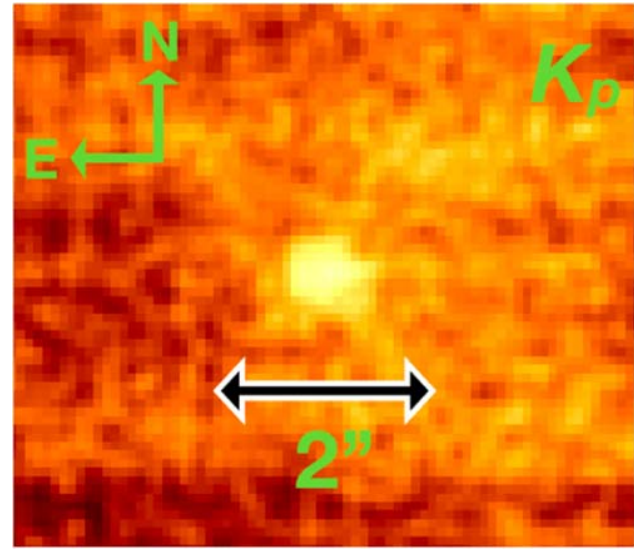

Figure 4. The $K_{p}$ image of $2 \mathrm{I}$ taken with the OSIRIS AO instrument on Keck I tracking at the sky motion rate of the comet. The image is a composite stack of four $60 \mathrm{~s} K_{p}$ exposures stacked on the position of the comet in each individual exposure. The image has been $4 \times 4$ binned, giving it a pixel scale of $0 . \prime 08$. The FWHM of the background stars measured perpendicular to the rate of motion of $2 \mathrm{I}$ is $\sim 0 . \prime 2$. The detection is PSF-like without an extended appearance or a tail visible in the image. The image has been Gaussian smoothed by 2 binned pixels.

inflection point in the light curve seen at distances $r_{h}<3$ au that tell us that additional water outgassing has turned on and started to dominate the activity of the object. This latter finding, of water outgassing dominance, again tells us that 2I appears to be acting like a normal solar system comet, as water is by far the most abundant ice found in solar system comets.

\subsection{Mass Loss}

Using the $g, r, i$, and $z$ photometry obtained by the ARC $3.5 \mathrm{~m}$ on 2019 September 12 UTC, we place estimates on 2I's Af $\rho$ parameter, a proxy for dust production rate (A'Hearn et al. 1984). We find $(A f \rho)_{g}=113 \pm 5 \mathrm{~cm},(A f \rho)_{r}=185 \pm 7 \mathrm{~cm}$, $(A f \rho)_{i}=223 \pm 8 \mathrm{~cm}$, and $(A f \rho)_{z}=180 \pm 8 \mathrm{~cm}$, typical values for solar system comets (A'Hearn et al. 1995; Kelley et al. 2013), implying an outgassing rate of $\sim 10^{27} \mathrm{~mol} \mathrm{~s}^{-1}$ (Fink \& Rubin 2012). The recently taken data from between 2019 September 11 UTC and 2019 December 20 UTC seen in Table 1 and Figure 5 show a brightening trend of $\sim 0.03$ mag day ${ }^{-1}$, consistent with the enhancement in brightness expected for the evolving viewing geometry of the comet according to the equation

$$
m_{V}=H_{\mathrm{abs}}+2.5 \log _{10}\left(r_{h} \Delta\right)+\Phi(\alpha),
$$

where $m_{V}$ is the apparent magnitude; $H_{\mathrm{abs}}$ is the absolute magnitude; $r_{h}$ is the heliocentric distance in au; $\Delta$ is the observercentric distance in au; $\Phi(\alpha)$ is a function describing the brightening of the comet, which we approximate with $\Phi(\alpha)=$ $-0.04 \alpha$ (Jewitt 1991); and $\alpha$ is the phase angle of the comet measured in degrees, appropriate for comets at smaller phase angles than $\sim 20^{\circ}$ (Bertini et al. 2017). We translate the $H_{\text {abs }}$ magnitude computed from Equation (1) into an effective cross section, $C$, in units of $\mathrm{km}^{2}$ within a $10,000 \mathrm{~km}$ aperture using 


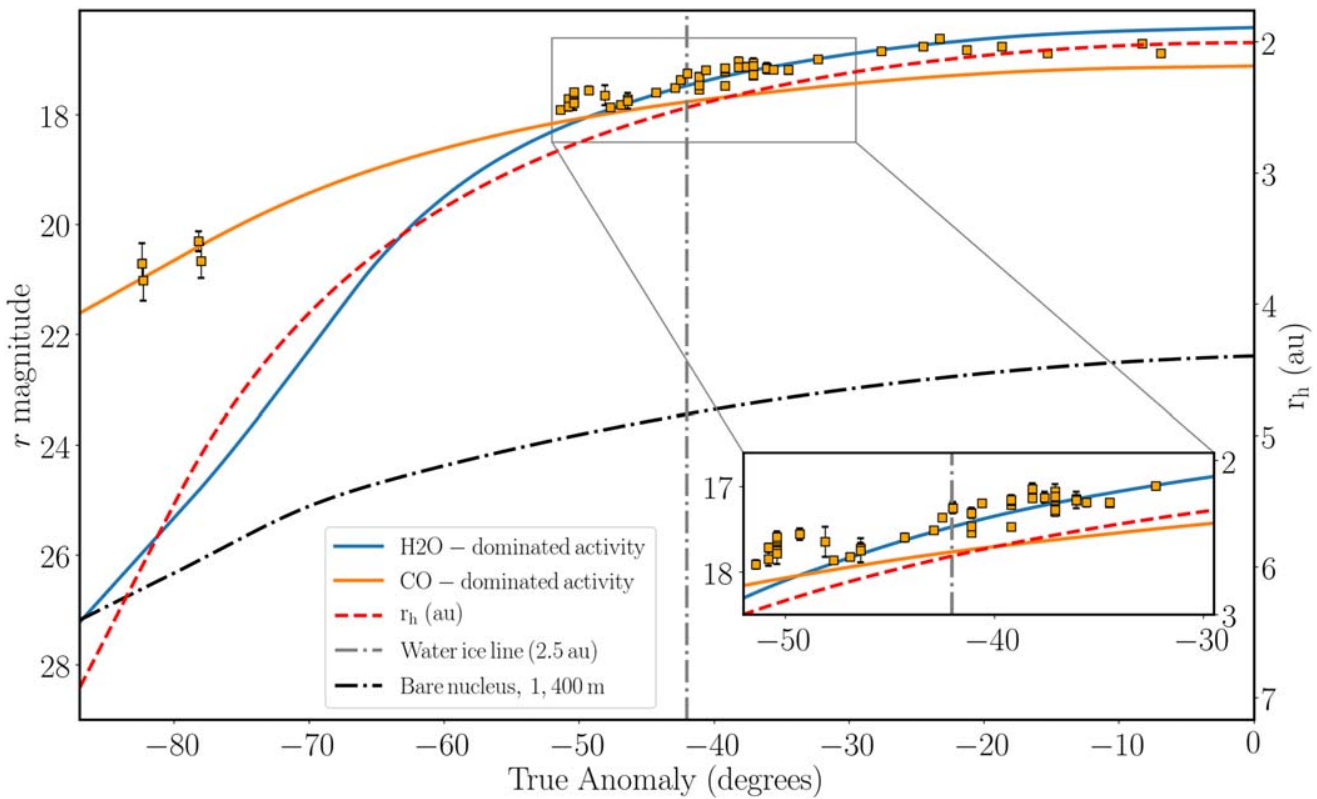

Figure 5. The $r$ magnitude of 2I as a function of the true anomaly using photometry translated to $r$ magnitudes for data taken between 2019 March 17 (Ye et al. 2020 ) and 2019 November 29 (this campaign) and tabulated in Table 1. The blue and orange lines are the predicted brightness as a function of true anomaly angle for $\mathrm{H}_{2} \mathrm{O}-$ and CO-dominated activity for a comet with a diameter of $1.4 \mathrm{~km}$ and $100 \%$ active surface area from the outgassing model of 2I from Fitzsimmons et al. (2019). The brightness prediction assumes a $5^{\prime \prime}$ aperture, comparable to the aperture size used to measure the brightness of 2I in this study. The dashed-dotted black line is the predicted brightness as a function of true anomaly angle assuming an inactive bare nucleus, a $1.4 \mathrm{~km}$ diameter, and a 0.04 albedo, the lower limit on the estimate of 2I's nucleus size from the detection of CN gas (Fitzsimmons et al. 2019). The red dashed line shows the heliocentric distance, $r_{h}$, as a function of true anomaly for 2I. The vertical gray dashed-dotted line is positioned on the true anomaly where $2 \mathrm{I}$ crosses the water-ice line at 2.5 au. True anomaly $=0^{\circ}$ corresponds to $2 \mathrm{I}^{\prime} \mathrm{s}$ perihelion passage on 2019 December 8 UTC.

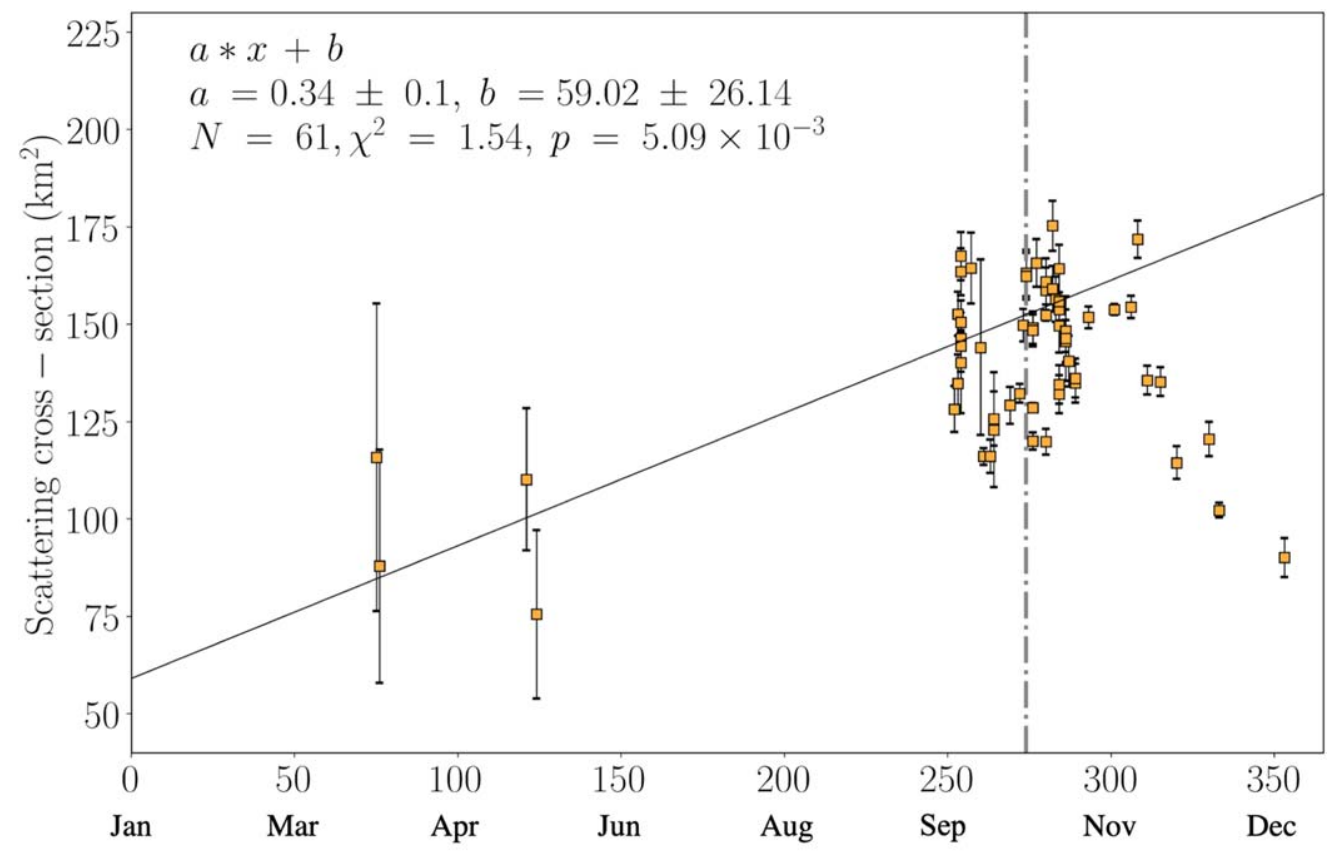

days since $2019-01-01$

Figure 6. Effective cross section of 2I calculated from Equation (2) as a function of days since 2019 January 1 UTC. The black line shows the minimized $\chi^{2}$ fit to the cross-section measurements, and the vertical dashed-dotted line corresponds to the date when 2I crossed the water-ice line at 2.5 au.

the formula

$$
C=1.5 \times 10^{6} p_{v}^{-1} 10^{-0.4 H}
$$

from Jewitt et al. (2016), where $p_{v}$ is the albedo of the comet, assumed to be 0.10, typical for comet dust (Jewitt \& Meech 1986;
Kolokolova et al. 2004). We caution that uncertainties of $H_{\text {abs }}$ inferred from Equation (1) are lower limits on the overall photometric uncertainty because they should also include a component from the phase function, which is unknown at the present time for $2 \mathrm{I}$. 
We plot the effective cross section over the baseline of available 2I photometry,including the ZTF prediscovery data taken in 2019 March and May, as seen in Figure 6. The median cross section from these data is $\sim 145 \mathrm{~km}^{2}$. A linear fit is applied to the data with the minimized $\chi^{2}$ fit corresponding to a slope of $0.34 \pm 0.10 \mathrm{~km}^{-2}$ day $^{-1}$, suggesting that the cross section doubled since the earliest observations from the ZTF prediscovery images in 2019 March 17 and will exceed $\sim 200 \mathrm{~km}^{2}$ by the time 2I reaches perihelion on 2019 December 8 UTC, assuming the slope is constant. We note that the data point corresponding to the 2019 November 29 UTC and 2019 December 20 UTC data may be due to 2I increasing in brightness at a slower rate than expected as the comet reaches perihelion, so we do not include it with our linear fit.

There appears to be a sudden, $\sim 50 \mathrm{~km}^{2}$ jump in the effective cross section between 2019 September 20 and 2019 October 3 UTC, as seen in Figure 6, corresponding to the drop in the overall trend for brightness seen in the light curve plotted in Figure 5 between true anomaly angles $-47^{\circ}$ and $-42^{\circ}$. As discussed in Section 3.3, the deviation in brightness may indicate a change in the comet's activity. The location of the vertical dashed-dotted line in Figure 6 indicates when 2I crossed the water-ice line, which is near an observed steep increase in the cross section, possibly connected to the sublimation of $\mathrm{H}_{2} \mathrm{O}$ discussed further in Section 3.3. There is also another, earlier $\sim 50 \mathrm{~km}^{2}$ jump in the cross section starting around the onset of our observations on 2019 September 10 UTC, though we caution that the variability can also be due to the large errors of the individual data points.

\subsection{Diameter Estimate}

A rough upper limit to the diameter of $2 \mathrm{I}$ of $\sim 5-10 \mathrm{~km}$ was found using our conventional ground-based observations, typically on the order of $\sim 1^{\prime \prime}$ resolution, similar to the size upper limit estimate of $\sim 8 \mathrm{~km}$ from Jewitt \& Luu (2019). Coma-subtraction techniques that remove the dust component from the total effective cross section of the comet (i.e., Fernández et al. 2013; Bauer et al. 2017) proved to be only partially effective due to the density of the coma at the resolution afforded by ground-based observations.

A more accurate upper limit can be inferred by measuring the effective cross section using high-resolution data from high-resolution ground-based AO and space-based observations from Keck (e.g., Marchis et al. 2006). Using a 0!.48 aperture with a contiguous median sky-subtraction annulus from 0 ". 48 to 0 ". 96 , we obtain $K p=19.63 \pm 0.09$. We use our visible and NIR colors determined for 2I to transform the $K_{p}$ magnitude measured in the OSIRIS images taken on 2019 October 4 UTC to $V=21.95 \pm 0.16$ from our combined VisNIR photometry and IRTF spectrum presented in Sections 3.1 and 3.2. We use the $V$ magnitude to calculate $H_{\text {abs }}=16.88 \pm 0.16$ using Equation (1) with the $r_{h}=2.48$ au, $\Delta=2.96$ au, and $\alpha=18^{\circ} .65$ that the comet had on 2019 October 4 UTC. As mentioned in Section 3.4, the uncertainty on the $H_{\text {abs }}$ calculation is a lower limit due to the unknown phase function of the comet.

We converted the $H_{\text {abs }}$ magnitude determined with the 0 ". 48 aperture into an effective cross section using Equation (2), resulting in an effective cross section of $2.65 \pm 0.39 \mathrm{~km}^{2}$, assuming an albedo equal to 0.1 , typical for comet dust, and resulting in a value of $6.63 \pm 0.97 \mathrm{~km}^{2}$, assuming an albedo equal to 0.04, typical for comet nuclei (Fernández et al. 2001;
Bauer et al. 2017). A higher albedo could also be used to calculate the cross section corresponding to an icy, more reflective composition (Yang et al. 2009), but the NIR spectra presented here, as well as additional NIR spectra (Yang et al. 2019), do not show strong evidence for the presence of ice in the coma of $2 \mathrm{I}$.

Using the equation $D=2 \sqrt{C / \pi}$ to calculate the diameter from $C$, we obtain the values $1.84 \pm 0.13$ and $2.90 \pm 0.21 \mathrm{~km}$ for $p v=0.1$ and 0.04 , respectively, implying a mass of $\lesssim 10^{12}$ $\mathrm{kg}$, assuming a comet nucleus density of $400 \mathrm{~kg} \mathrm{~m}^{-3}$ (e.g., Pätzold et al. 2016). In addition to the advantages of using higher-resolution AO imaging compared to conventional ground-based observations, observing comets in longer wavelengths such as the $K_{p}$ band has the advantage of avoiding much of the scattered light from micron-sized dust that is more prevalent in visible wavelengths. This effect of using less dustcontaminated wavelengths in the photometry of comets has already been demonstrated to produce robust diameter estimates of comets, even at spatial resolutions approaching or worse than in the $K_{p} \mathrm{AO}$ images presented here (Fernández et al. 2013; Bauer et al. 2017).

We caution that the estimates of the nucleus size are strictly rough upper limits (Bolin 2020). Profiles through the imagery, especially the high spatial resolution Keck images, do not show a discernible signal due to a point-source nucleus, arguing for an object dominated in brightness by scattered light from its surrounding coma (e.g., Jewitt \& Luu 2019; Kim et al. 2020) and suggesting a small (less than a few $\mathrm{km}$ diameter) nucleus at the 2.9 au distance at which 2I was observed by Keck.

We thus resort to estimating its nucleus size in three different ways: (1) a very optimistic method that includes all the flux detected in the central PSF, in order to determine a hard upper limit for the nucleus' size; (2) a more realistic method that involves extrapolating the run of coma brightness versus distance from the nucleus into the central PSF, allowing us to model the coma in the entire image and then remove it; and (3) a hybrid approach whereby we take the flux from method (1) and modify it for known observations of hyperactive solar system comets.

The first method yields an object with a diameter of $\sim 3 \mathrm{~km}$, giving us a hard upper limit to 2I's size; it cannot be on the order of $20 \mathrm{~km}$ diameter or greater, as some initial estimates have stated. The second method is much more constraining, as we do not detect a nucleus residual after modeling and removing the coma (assuming a stellar PSF derived from cuts through highly trailed stars perpendicular to the trailing direction). Adopting a $2 \sigma$ upper limit from the noise level of the coma removal ( $\sim 10 \%$ of the central PSF flux), we find an upper limit to the $2 \mathrm{I}$ diameter of $\sim 1.4 \mathrm{~km}$, similar to the prediction of 2I's size by the thermal model presented in Fitzsimmons et al. (2019). The third method takes note of the fact that a small 2I nucleus size implies a very high outgassing rate per unit $\mathrm{km}^{2}$ of nucleus surface area, a phenomenon seen for "hyperactive" solar system comets like 103P Hartley 2 (Lisse et al. 2009; A'Hearn et al. 2011; Harker et al. 2018) and $46 \mathrm{P} /$ Wirtannen (Lis et al. 2019) to be due to large amounts of ice-rich dust expulsion into the surrounding coma, greatly increasing the active surface area receiving solar insolation. Using the ratio of $\sim 4: 1$ coma:nucleus surface brightness seen for comet 103P during the Deep Impact mission in situ flyby, we can scale the total flux in the central PSF by a factor of $1 /(1+4)=0.2$ and then proceed as if we have measured the 
nucleus's flux. Doing so, we arrive again at an estimated nucleus diameter upper limit of $\sim 1.4 \mathrm{~km}$, similar to nucleus measurements from high-resolution space-based observations (Jewitt et al. 2020).

\section{Discussion and Conclusions}

The second ISO, 2I, seems on all accounts like an ordinary comet compared to the comets of the solar system, though it is depleted in some chemical species relative to solar system comets (Kareta et al. 2020; Opitom et al. 2019; Bannister et al. 2020) and has an excess of CO (Bodewits et al. 2020; Cordiner et al. 2020). If it were not for its significantly hyperbolic orbit, 2I probably would not have warranted an in-depth scientific investigation. However, given its special status as a comet of extrasolar origin, it presents a unique opportunity to study the cometary components of other star systems, since a likely outcome of the evolution of planetary systems is the ejection of many cometary bodies (Raymond et al. 2018a, 2018b). In our own solar system, the comet population is a record of its formation properties and evolution (Morbidelli \& Nesvorny 2020), so by studying objects that were ejected from their home systems, like 1I and 2I, we can directly observe the consequences of planetary system evolution.

One of the salient properties of $2 \mathrm{I}$ is that it contains significant amounts of volatiles such as $\mathrm{CN}$ and $\mathrm{C}_{2}$ gas (Fitzsimmons et al. 2019; Kareta et al. 2020; Opitom et al. 2019), and there is evidence in this work from the photometry presented in Section 3.3 that the comet also contains $\mathrm{H}_{2} \mathrm{O}$, unlike the super-rich $\mathrm{CO} / \mathrm{N} 2 / \mathrm{CH} 4, \mathrm{H}_{2} \mathrm{O}$-depleted comet C/2016 R2 (Cochran \& McKay 2018; McKay et al. 2020). Instead, it is acting like an Oort cloud comet on a megayearperiod orbit like C/1995 O1 (Hale-Bopp), C/2013 S1 (ISON), or C/2017 K2 (Jewitt et al. 2017a; Meech et al. 2017), which commonly demonstrate outgassing abundances of $\mathrm{CO}$ with respect to water in the $0.2 \%-20 \%$ range (Bockelée-Morvan et al. 2004). The presence of moderately abundant $\mathrm{CO}$ and $\mathrm{H}_{2} \mathrm{O}$ on 2I (Ye et al. 2020 and this work) suggests that while 2I has not been heated so thoroughly by its home Sun (as solar system Jupiter family comets and likely 1I have), it could have been ejected from its home system or placed into its star's equivalent of the solar system's Oort cloud more than a few Myr of its formation after its home system's protoplanetary disk midplane had cleared enough to heat its surface above $30 \mathrm{~K}$ (Lisse et al. 2019). This assumes that in comparison with the solar system comet $\mathrm{C} / 2016 \mathrm{R} 2$, has never been heated above $20 \mathrm{~K}$ before encountering the Sun, where it is in the process of losing its hypervolatiles but not its $\mathrm{H}_{2} \mathrm{O}$ ice due to hypervolatile supercooling (Biver et al. 2018; Lisse et al. 2019). Additionally, the host star of 2I may have a higher stellar iron abundance that has been shown to have an effect on the waterice fraction solid building blocks in the protoplanetary disk favoring a higher concentration of $\mathrm{CO} / \mathrm{CO}_{2}$ relative to water ice (Bitsch \& Battistini 2020).

Compared to 2I, 1I had only marginal levels of activity. The activity of 1I was not seen in direct imaging of the comet or in its spectra (Meech et al. 2017; Fitzsimmons et al. 2018), only being evident via detailed astrometry of the small trajectory deviations from inertial-solar gravitation caused by low levels of outgassing (Micheli et al. 2018). So, if it was actively outgassing, its coma was very faint and below the noise level in any of the detection images, including imaging from HST. One explanation for the lack of activity of $1 \mathrm{I}$ is that it had a mantle built up by cosmic-ray bombardment during its interstellar travel, trapping its volatiles inside its structure (Fitzsimmons et al. 2018). On the other hand, the specific outgassing rate per unit body surface area implied by the nongraviational force model of Micheli et al. (2018) is on the upper bound of Jupiter family comet activity (Fernández et al. 2013). With an $\sim 250 \mathrm{~m}$ diameter (Meech et al. 2017; Trilling et al. 2018), 1I was small compared to the typical $\mathrm{km}$ scale for a JFC comet, so it took very little force from outgassing to significantly accelerate it.

The activity of 2I can possibly be used to distinguish between the "large" or "small" size estimates for 2I discussed in Section 3.5, especially in comparison to 1I, by constraining the effect of nongravitational forces due to outgassing on its orbital trajectory. Moderate nongravitational force parameters have been measured for the orbit of 2I in prediscovery data when the comet's activity was weaker (Ye et al. 2020), as has been done for solar system comets (e.g., Moreno et al. 2017). If 2I has a similar size as 1I, then its small total volume and mass mean that it could also be accelerated much more by nongravitationally outgassing jet forces compared to 1I, given the apparent much larger outgassing rate for 2I than 1I. However, if $2 \mathrm{I}$ is much larger than $1 \mathrm{I}$ where the mass ratio between 2 I and 1 I scales as $(3 \mathrm{~km} / 0.25 \mathrm{~km})^{3}, \sim 1000$ times more massive than 1 I, then 2 I can be outgassing $\sim 1000$ times more than 1I and still suffer the same amount of jet acceleration. Thus, monitoring the astrometric position of 2I throughout the next few months will be critical for understanding the size regime of 2I's nucleus as its activity grows and its orbit can be potentially more affected by nongravitational forces.

Other estimates of size distribution for the ISO population have included upper limits on both the nondetection of ISOs (Engelhardt et al. 2017) and the sole detection of 1I (Trilling et al. 2017; Raymond et al. 2018b). We estimate the size distribution of the ISO population updated with the detection of $2 \mathrm{I}$ and the upper limit on its diameter from high-resolution images. We calculate the number of $250 \mathrm{~m}$ ISOs to be $\sim 13$ objects within 3 au of the Sun by scaling the density of $250 \mathrm{~m}$ ISOs, one ISO within 1 au of the Sun at any given time (Meech et al. 2017), to a sphere of radius $3 \mathrm{au}$, accounting for the gravitational focusing of the Sun and assuming a velocity at infinity of $32 \mathrm{~km} \mathrm{~s}^{-1}$ for ISOs as for comet 2I. Assuming a slightly lower velocity at infinity of $26 \mathrm{~m} \mathrm{~s}^{-1}$ as for 1I does not significantly change the results.

We calculate the relative number of $250 \mathrm{~m}$ diameter ISOs like $1 \mathrm{I}$ to $1.4 \mathrm{~km}$ ISOs like 2I by the fraction of time 2I was observable within 3 au over the total survey lifetime of the past $15 \mathrm{yr}$. We consider $15 \mathrm{yr}$ to be the amount of time that the search for objects like 2I by amateur astronomers was active due to the difficulty in obtaining sensitive CCD cameras at the consumer level before this time (Copandean et al. 2017). This translates into $13 \pm 13$ ISOs with a diameter of $\sim 250 \mathrm{~m}$ to $\sim 4 \times 10^{-2} \pm 4 \times 10^{-2}$ ISOs with a diameter of $\sim 2-3 \mathrm{~km}$ within 3 au of the Sun, where the uncertainties are estimated from the allowable range in number of 1I-like and 2I-like objects assuming Poissonian statistics. The resulting cumulative size distribution inferred from the ratio of the number of $250 \mathrm{~m}$ objects to $1.5-3 \mathrm{~km}$ objects is shown in Figure 7 . The slope of the cumulative size distribution is $\sim-3.38 \pm 1.18$, which is comparable to the cumulative size distribution slope of collisionally evolved solar system bodies (Dohnanyi 1969) and 


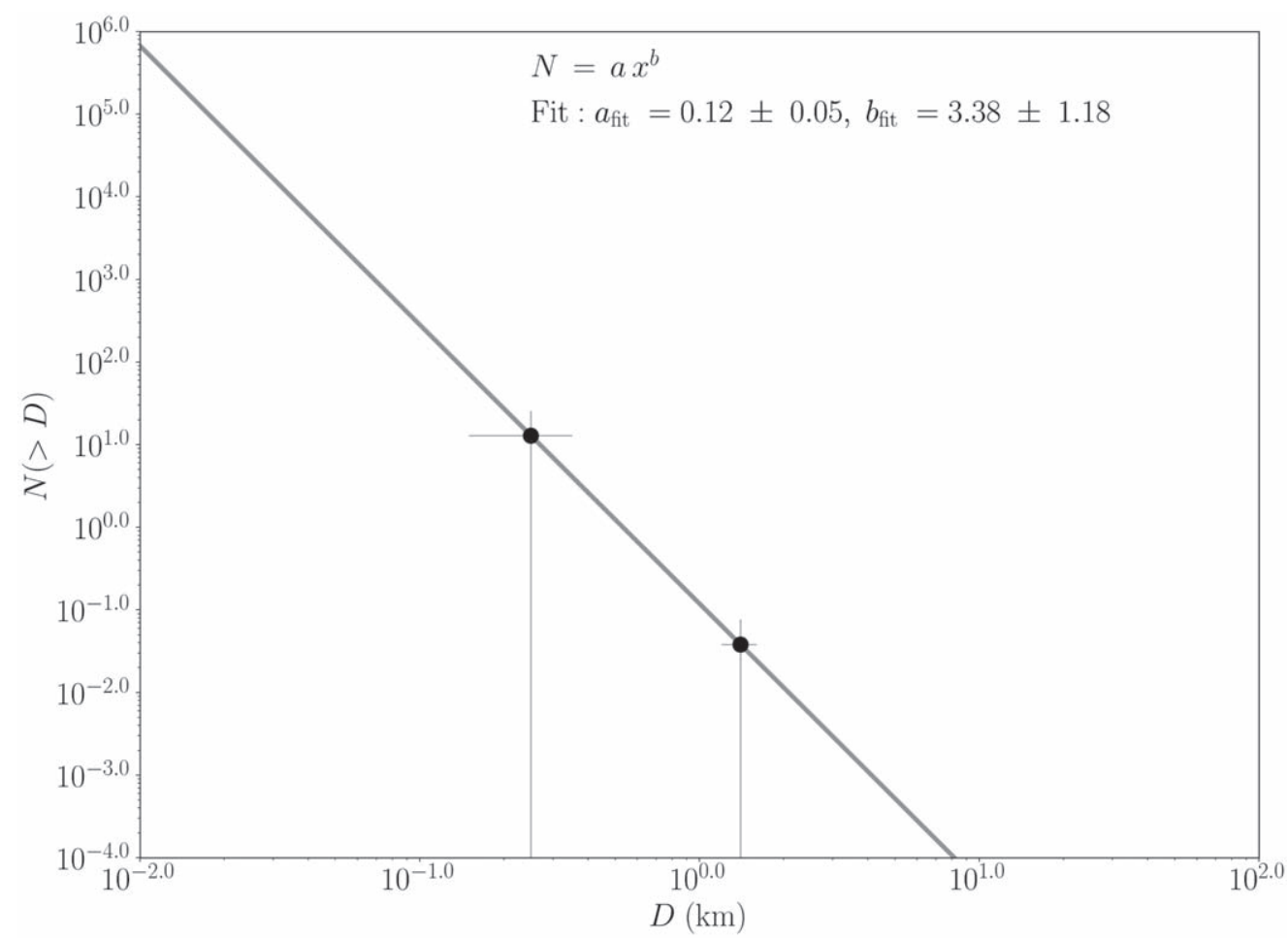

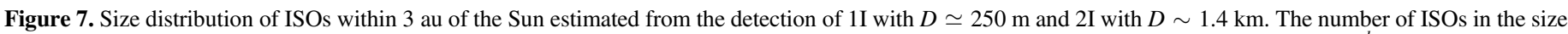

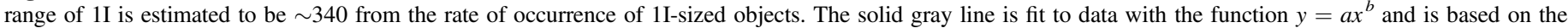

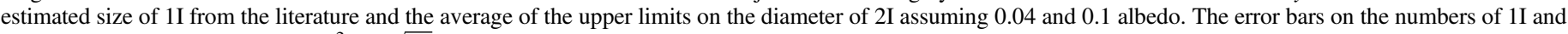

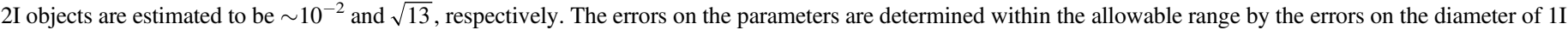
and 2I (Trilling et al. 2018, this work) and the number of 1I-like and 2I-like objects assuming Poissonian statistics.

comets measured in the kilometer diameter range (Meech \& Svoren 2004; Fernández et al. 2013; Boe et al. 2019a).

The size estimates we have derived for 2I above bear on the question of why "asteroidal" object 1I/Oumuamua was detected before an active, bright object like cometary 2I. Cognizant of the dangers of extrapolating size distributions and population statistics from a sample of $N=2$ purportedly related objects, we do so here because these arguments will likely be valuable in the fullness of time as we collect more and more detections of ISOs over the next decades. Naively, one would have expected active, $\sim 3$ mag brighter 2I-like objects to have been detected first modulo selection effects (e.g., Jedicke et al. 2016; Vokrouhlický et al. 2017) because they can be seen out to much farther distances (the detectability distance scales as the object's $D$, so the volume of space it can be detected in goes as the objects $D^{2}$ ). If 2I is substantially bigger than 1I, then for a steep enough ISO size distribution (slope steeper than -3 ), there can be many more 1Is in the volume of space than 2I-like objects, enough so that 1I-like objects will be seen more frequently. For a size distribution scaling with $\sim D^{-3}$ and $D_{1 \mathrm{I}} \simeq 250 \mathrm{~m}, D_{2 \mathrm{I}} \simeq 2.0 \mathrm{~km}$, there would be several hundred 1I-like objects for every one 2I in a given volume of space, overwhelming the 100 times larger volume that a $2 \mathrm{~km}$ diameter 2I-like object could be detected in.

Recent evidence suggests that the slope of active comets goes from steeper to shallower at a transition boundary of $D \sim 3 \mathrm{~km}$ (see Figure 5 of Boe et al. 2019a). In the $D \lesssim 3$ range, the cumulative size distribution slope is significantly shallower than objects $\gtrsim 3 \mathrm{~km}$ in size, which seems to contradict the slope of the size distribution that we measure for the ISOs. However, work in preparation on the size distribution of inactive comets in the subkilometer range shows a steeper size distribution than compared to subkilometer active comets and more closely resembles the slope of the ISO size distribution from this work (Boe et al. 2019b). Assuming that the properties of the size distributions of active and inactive comets in the solar system are shared with those in extrasolar systems, 1I may be a representative of the inactive comet population, given its lack of activity, and may come from a population with a steep size distribution, explaining its small size. In addition, a steeper size distribution for ISOs may indicate more ISOs being on retrograde orbits as they pass through the Solar System (Marčeta \& Novaković 2020). The observed activity of 2I suggests that it comes from an active comet population that has a shallower size distribution which would be consistent with 2I having a larger size than 1I. Alternatively, if $2 \mathrm{I}$ actually has a size comparable to $1 \mathrm{I}$, its apparent activity might indicate that it comes from a shallower size distribution compared to the size distribution of inactive objects. Thus, the relative numbers of active and inactive objects from correspondingly shallower and steeper size distributions may explain the relative frequency of inactive objects like 1I compared to inactive objects.

The ISO size distribution that we are observing may be a hybrid of both active and inactive comet populations from the ensemble of comet-ejecting extrasolar systems producing a mixed active and inactive observed ISO population resulting in a size distribution slope steeper than $\sim-3$. Although the error bars from our measurement of the ISO size distribution slope from the occurrence of 1I and 2I are large, future observations of ISOs could refine the measurement of the slope. A shallower slope would be more consistent with production from a 
population in collisional equilibrium (e.g., Dohnanyi 1969), while a steeper slope may indicate that the ISO population is fed partially by additional fragmentation events, such as tidal disruption (Bolin et al. 2018; Raymond et al. 2018a; Zhang \& Lin 2020). In any case, the existence of subkilometer interstellar comets like 1I suggests that the size distribution of objects in extrasolar Kuiper Belts, the progenitors of extrasolar comets, is not truncated at $1-2 \mathrm{~km}$, challenging the claim that the size distribution of objects in the solar system's Kuiper Belt is effectively truncated at $1-2 \mathrm{~km}$ in diameter (Singer et al. 2019). The arrival of additional ISOs will provide further constraints on their physical properties and size distribution, enhancing our understanding of comets in extrasolar systems.

We would like to thank the anonymous reviewers for helpful comments in substantially improving the manuscript.

This work was supported by the GROWTH project, funded by the National Science Foundation under PIRE grant No. 1545949.

Our work includes observations obtained with the Apache Point Observatory $3.5 \mathrm{~m}$ telescope, which is owned and operated by the Astrophysical Research Consortium. We thank the Director (Nancy Chanover) and Deputy Director (Ben Williams) of the Astrophysical Research Consortium (ARC) $3.5 \mathrm{~m}$ telescope at Apache Point Observatory for their enthusiastic and timely support of our Director's Discretionary Time (DDT) proposals. We also thank Russet McMillan, Ted Rudyk, Candace Gray, Jack Dembicky, and the rest of the APO technical staff for their assistance in performing the observations on the same day our DDT proposals were submitted.

Based on observations obtained with the Samuel Oschin Telescope 48 inch and the 60 inch telescope at the Palomar Observatory as part of the Zwicky Transient Facility project. The ZTF is supported by the National Science Foundation under grant No. AST-1440341 and a collaboration including Caltech, IPAC, the Weizmann Institute for Science, the Oskar Klein Center at Stockholm University, the University of Maryland, the University of Washington, Deutsches Elektronen-Synchrotron and Humboldt University, Los Alamos National Laboratories, the TANGO Consortium of Taiwan, the University of Wisconsin at Milwaukee, and Lawrence Berkeley National Laboratories. Operations are conducted by COO, IPAC, and UW.

Some of the data presented herein were obtained at the W. M. Keck Observatory, which is operated as a scientific partnership among the California Institute of Technology, the University of California, and the National Aeronautics and Space Administration. The Observatory was made possible by the generous financial support of the W. M. Keck Foundation.

We thank Jim Lyke and Carlos Alvarez for guiding the planning and executing the AO observation with the OSIRIS instrument on Keck I.

C.-C.N. is thankful for the funding from MoST grant 1042923-M-008-004-MY5.

The work of D.S. was carried out at the Jet Propulsion Laboratory, California Institute of Technology, under a contract with NASA.

SED Machine is based upon work supported by the National Science Foundation under grant No. 1106171.
This publication has made use of data collected at Lulin Observatory, partly supported by MoST grant 108-2112-M008-001.

The results presented in this paper are based in part on observations collected with the Liverpool Telescope, which is operated on the island of La Palma by Liverpool John Moores University in the Spanish Observatorio del Roque de los Muchachos of the Instituto de Astrofisica de Canarias with financial support from the UK Science and Technology Facilities Council.

Visiting Astronomer at the Infrared Telescope Facility, which is operated by the University of Hawaii under contract NNH14CK55B with the National Aeronautics and Space Administration.

The authors wish to recognize and acknowledge the very significant cultural role and reverence that the summit of Maunakea has always had within the indigenous Hawaiian community. We are most fortunate to have the opportunity to conduct observations from this mountain.

F.M. is supported by an appointment to the NASA Postdoctoral Program at the Jet Propulsion Laboratory, administered by the Universities Space Research Association under contract with NASA.

The authors would like to thank E. Turner, B. Draine, S. Tremaine, and M. Mac-Low for many useful discussions concerning the nature and provenance of $1 \mathrm{I}$ and $2 \mathrm{I}$. In addition, the authors would like to thank R. Jedicke and G. Helou for helpful discussion on the size distribution of comets. We would also like to thank J. Bauer and Y. Fernandez for fruitful discussion on comet nuclei.

The authors would like to thank the astrophysics masters students of the Universite Côte d'Azur who recorded the data taken by the C2PU telescope at Observatoire de la Côte d'Azur, Calern observing site.

Facilities: Astrophysical Research Consortium 3.5 m, C2PU Omicron telescope, Bisei Observatory $101 \mathrm{~cm}$, Lulin Optical Telescope, Keck:I (LRIS, OSIRIS), Liverpool Telescope, Mount Laguna Observatory 40 inch, NASA/Infrared Telescope Facility, Zwicky Transient Facility, SED Machine.

\section{ORCID iDs}

Bryce T. Bolin (10 https://orcid.org/0000-0002-4950-6323 Carey M. Lisse (ib https://orcid.org/0000-0002-9548-1526

Mansi M. Kasliwal (i) https://orcid.org/0000-0002-5619-4938 Robert Quimby (iD https://orcid.org/0000-0001-9171-5236 Chris M. Copperwheat (i) https://orcid.org/0000-00017983-8698

Kevin B. Burdge (DD https://orcid.org/0000-0002-7226-836X Michael Coughlin (iD https://orcid.org/0000-0002-8262-2924 Christoffer Fremling (iD https:// orcid.org/0000-00024223-103X

Ryosuke Itoh (iD https://orcid.org/0000-0002-1183-8955 Michael Koss (ib https://orcid.org/0000-0002-7998-9581 Frank J. Masci (i) https://orcid.org/0000-0002-8532-9395 Eric E. Mamajek (iD https://orcid.org/0000-0003-2008-1488 Federico Marocco (1D https://orcid.org/0000-0001-7519-1700 Michael L. Sitko (1) https://orcid.org/0000-0003-1799-1755 Daniel Stern (D) https://orcid.org/0000-0003-2686-9241 Lin Yan (ib https://orcid.org/0000-0003-1710-9339 Igor Andreoni (iD https://orcid.org/0000-0002-8977-1498 Varun Bhalerao (i) https://orcid.org/0000-0002-6112-7609 Dennis Bodewits (ib https://orcid.org/0000-0002-2668-7248 
Eric C. Bellm (16) https://orcid.org/0000-0001-8018-5348 Nadejda Blagorodnova (10 https://orcid.org/0000-00030901-1606

Derek Buzasi (다 https://orcid.org/0000-0002-1988-143X S. Bradley Cenko (1) https://orcid.org/0000-0003-1673-970X Chan-Kao Chang (i) https://orcid.org/0000-0003-1656-4540 Dmitry A. Duev (iD https://orcid.org/0000-0001-5060-8733 Shrinivas R. Kulkarni ii https://orcid.org/0000-00015390-8563

Thomas Kupfer (10) https://orcid.org/0000-0002-6540-1484 Chow-Choong Ngeow (i) https://orcid.org/0000-00018771-7554

Reed Riddle (1) https://orcid.org/0000-0002-0387-370X Jesper Sollerman (1) https://orcid.org/0000-0003-1546-6615 Maayane T. Soumagnac (1) https://orcid.org/0000-00016753-1488

\section{References}

A'Hearn, M. F., Belton, M. J. S., Delamere, W. A., et al. 2011, Sci, 332, 1396 A'Hearn, M. F., Millis, R. C., Schleicher, D. O., Osip, D. J., \& Birch, P. V. 1995, Icar, 118, 223

A'Hearn, M. F., Schleicher, D. G., Millis, R. L., Feldman, P. D., \& Thompson, D. T. 1984 , AJ, 89, 579

Bannister, M. T., Opitom, C., Fitzsimmons, A., et al. 2020, arXiv:2001.11605 Bannister, M. T., Schwamb, M. E., Fraser, W. C., et al. 2017, ApJL, 851, L38 Bauer, J. M., Grav, T., Fernández, Y. R., et al. 2017, AJ, 154, 53

Bellm, E. C., Kulkarni, S. R., Barlow, T., et al. 2019a, PASP, 131, 068003

Bellm, E. C., Kulkarni, S. R., Graham, M. J., et al. 2019b, PASP, 131, 018002

Bertini, I., la Forgia, F., Tubiana, C., et al. 2017, MNRAS, 469, S404

Bitsch, B., \& Battistini, C. 2020, A\&A, 633, A10

Biver, N., Bockelée-Morvan, D., Paubert, G., et al. 2018, A\&A, 619, A127

Blagorodnova, N., Neill, J. D., Walters, R., et al. 2018, PASP, 130, 035003

Bockelée-Morvan, D., Crovisier, J., Mumma, M. J., \& Weaver, H. A. 2004, in Comets II, ed. M. C. Festou, H. U. Keller, \& H. A. Weaver (Tucson, AZ: Univ. Arizona Press), 391

Bockelée-Morvan, D., Rinaldi, G., Erard, S., et al. 2017, MNRAS, 469, S443 Bodewits, D., Noonan, J. W., Feldman, P. D., et al. 2020, NatAs, in press

Boe, B., Jedicke, R., Meech, K. J., et al. 2019a, Icar, 333, 252

Boe, B., Jedicke, R., Wiegert, P., et al. 2019b, EPSC-DPS Joint Meeting, 2019, EPSC-DPS2019-626

Bolin, B. T. 2020, arXiv: 1912.07386

Bolin, B. T., Weaver, H. A., Fernandez, Y. R., et al. 2018, ApJL, 852, L2

Bus, S. J., \& Binzel, R. P. 2002, Icar, 158, 146

Chambers, K. C., Magnier, E. A., Metcalfe, N., et al. 2016, arXiv: 1612.05560

Chen, C. H., Sargent, B. A., Bohac, C., et al. 2006, ApJS, 166, 351

Cochran, A. L., \& McKay, A. J. 2018, ApJL, 854, L10

Copandean, D., Vaduvescu, O., \& Gorgan, D. 2017, in 13th IEEE Int. Conf. Intelligent Computer Communication and Processing, ed. R. Potolea \& R. Razvan Slavescu (Piscataway, NJ: IEEE), 377

Cordiner, M. A., Milam, S. N., Biver, N., et al. 2020, NatAs, in press de León, J., Licandro, J., Serra-Ricart, M., et al. 2019, RNAAS, 3, 131 DeMeo, F. E., Binzel, R. P., Slivan, S. M., \& Bus, S. J. 2009, Icar, 202, 160 DeMeo, F. E., \& Carry, B. 2013, Icar, 226, 723

Dohnanyi, J. S. 1969, JGR, 74, 2531

Engelhardt, T., Jedicke, R., Vereš, P., et al. 2017, AJ, 153, 133

Feldman, P. D., Cochran, A. L., \& Combi, M. R. 2004, in Comets II, ed. M. C. Festou, H. U. Keller, \& H. A. Weaver (Tucson, AZ: Univ. Arizona Press), 425

Fernández, Y. R., Jewitt, D. C., \& Sheppard, S. S. 2001, ApJL, 553, L197

Fernández, Y. R., Kelley, M. S., Lamy, P. L., et al. 2013, Icar, 226, 1138

Fink, U., \& Rubin, M. 2012, Icar, 221, 721

Fitzsimmons, A., Hainaut, O., Meech, K. J., et al. 2019, ApJL, 885, L9

Fitzsimmons, A., Snodgrass, C., Rozitis, B., et al. 2018, NatAs, 2, 133

Flewelling, H. A., Magnier, E. A., Chambers, K. C., et al. 2016, arXiv:1612. 05243

Graham, M. J., Kulkarni, S. R., Bellm, E. C., et al. 2019, PASP, 131, 078001

Hanuš, J., Delbo, M., Alí-Lagoa, V., et al. 2018, Icar, 299, 84

Harker, D. E., Woodward, C. E., Kelley, M. S. P., \& Wooden, D. H. 2018, AJ, 155,199

Huehnerhoff, J., Ketzeback, W., Bradley, A., et al. 2016, Proc. SPIE, 9908, $99085 \mathrm{H}$
Hughes, D. W. 1990, QJRAS, 31, 69

Hui, M.-T., Ye, Q.-Z., Föhring, D., Hung, D., \& Tholen, D. J. 2020, arXiv:2003.14064

Ivezić, Ž, Lupton, R. H., Jurić, M., et al. 2002, AJ, 124, 2943

Ivezić, Ž, Tabachnik, S., Rafikov, R., et al. 2001, AJ, 122, 2749

Jedicke, R., Bolin, B., Granvik, M., \& Beshore, E. 2016, Icar, 266, 173

Jewitt, D. 1991, in IAU Coll. 116: Comets in the post-Halley Era 167, Astrophysics and Space Science Library, ed. R. L. Newburn, Jr., M. Neugebauer, \& J. Rahe, 19

Jewitt, D., Hsieh, H., \& Agarwal, J. 2015, in Asteroids IV, ed. P. Michel, F. E. DeMeo, \& W. F. Bottke (Tucson, AZ: Univ. Arizona Press), 221

Jewitt, D., Hui, M.-T., Kim, Y., et al. 2020, ApJL, 888, L23

Jewitt, D., Hui, M.-T., Mutchler, M., et al. 2017a, ApJL, 847, L19

Jewitt, D., \& Luu, J. 2019, ApJL, 886, L29

Jewitt, D., Luu, J., Rajagopal, J., et al. 2017b, ApJL, 850, L36

Jewitt, D., \& Meech, K. J. 1986, ApJ, 310, 937

Jewitt, D., Mutchler, M., Weaver, H., et al. 2016, ApJL, 829, L8

Jordi, K., Grebel, E. K., \& Ammon, K. 2006, A\&A, 460, 339

Jurić, M., Ivezić, Ž, Lupton, R. H., et al. 2002, AJ, 124, 1776

Kareta, T., Andrews, J., Noonan, J. W., et al. 2020, ApJL, 889, L38

Kasliwal, M. M., Cannella, C., Bagdasaryan, A., et al. 2019, PASP, 131, 038003

Keller, H. U., Mottola, S., Hviid, S. F., et al. 2017, MNRAS, 469, S357

Kelley, M. S., Fernández, Y. R., Licandro, J., et al. 2013, Icar, 225, 475

Kim, Y., Jewitt, D., Mutchler, M., et al. 2020, ApJL, 895, L34

Kinoshita, D., Chen, C.-W., Lin, H.-C., et al. 2005, ChJAA, 5, 315

Kolokolova, L., Hanner, M. S., Levasseur-Regourd, A.-C., \& Gustafson, B. A S. 2004, in Comets II, ed. M. C. Festou, H. U. Keller, \& H. A. Weaver (Tucson, AZ: Univ. Arizona Press), 577

Larkin, J., Barczys, M., Krabbe, A., et al. 2006, Proc. SPIE, 6269, 62691A

Li, J.-Y., Besse, S., A'Hearn, M. F., et al. 2013, Icar, 222, 559

Li, J.-Y., Samarasinha, N. H., Kelley, M. S. P., et al. 2016, ApJL, 817, L23

Lis, D. C., Bockelée-Morvan, D., Güsten, R., et al. 2019, A\&A, 625, L5

Lisse, C. M., Fernandez, Y. R., Reach, W. T., et al. 2009, PASP, 121, 968

Lisse, C. M., Sitko, M. L., Marengo, M., et al. 2017, AJ, 154, 182

Lisse, C. M., Wyatt, M. C., Chen, C. H., et al. 2012, ApJ, 747, 93

Lisse, C. M., Young, L. A., Cruikshank, D., et al. 2019, AGU Meeting, 2019, P33I-3540

MacGregor, M. A., Weinberger, A. J., Nesvold, E. R., et al. 2019, ApJL, 877, L32

Marčeta, D., \& Novaković, B. 2020, MNRAS, in press

Marchis, F., Kaasalainen, M., Hom, E. F. Y., et al. 2006, Icar, 185, 39

Masci, F. J., Laher, R. R., Rusholme, B., et al. 2019, PASP, 131, 018003

McKay, A. J., Cochran, A. L., Dello Russo, N., \& DiSanti, M. 2020, ApJL, 889, L10

Meech, K. J. 2017, RSPTA, 375, 20160247

Meech, K. J., \& Svoren, J. 2004, in Comets II, ed. M. C. Festou, H. U. Keller, \& H. A. Weaver (Tucson, AZ: Univ. Arizona Press), 317

Meech, K. J., Weryk, R., Micheli, M., et al. 2017, Natur, 552, 378

Meng, H. Y. A., Su, K. Y. L., Rieke, G. H., et al. 2014, Sci, 345, 1032

Micheli, M., Farnocchia, D., Meech, K. J., et al. 2018, Natur, 559, 223

Morbidelli, A., \& Nesvorny, D. 2020, in The Trans-Neptunian Solar System, ed. D. Prialnik, M. Antoinetta Barucci, \& L. Young (Amsterdam: Elsevier) Moreno, F., Pozuelos, F. J., Novaković, B., et al. 2017, ApJL, 837, L3

Ofek, E. O. 2012, ApJ, 749, 10

Opitom, C., Fitzsimmons, A., Jehin, E., et al. 2019, A\&A, 631, L8

Paganini, L., Mumma, M. J., Villanueva, G. L., et al. 2014, ApJ, 791, 122

Pätzold, M., Andert, T., Hahn, M., et al. 2016, Natur, 530, 63

Protopapa, S., sunshine, J. M., Feaga, L. M., et al. 2014, Icar, 238, 191

Rappaport, S., Vanderburg, A., Jacobs, T., et al. 2018, MNRAS, 474, 1453

Raymond, S. N., Armitage, P. J., \& Veras, D. 2018a, ApJL, 856, L7

Raymond, S. N., Armitage, P. J., Veras, D., Quintana, E. V., \& Barclay, T. 2018b, MNRAS, 476, 3031

Rayner, J. T., Toomey, D. W., Onaka, P. M., et al. 2003, PASP, 115, 362 Rigault, M., Neill, J. D., Blagorodnova, N., et al. 2019, A\&A, 627, A115 Schwamb, M. E., Fraser, W. C., Bannister, M. T., et al. 2019, ApJS, 243, 12 Singer, K. N., McKinnon, W. B., Gladman, B., et al. 2019, Sci, 363, 955 Skrutskie, M. F., Cutri, R. M., Stiening, R., et al. 2006, AJ, 131, 1163 Smith, C. E., \& Nelson, B. 1969, PASP, 81, 74

Snodgrass, C., Agarwal, J., Combi, M., et al. 2017, A\&ARv, 25, 5 Solontoi, M., Ivezić, Ž, Jurić, M., et al. 2012, Icar, 218, 571

Steele, I. A., Smith, R. J., Rees, P. C. T., et al. 2004, Proc. SPIE, 5489, 679 Su, K. Y. L., Jackson, A. P., Gáspár, A., et al. 2019, AJ, 157, 202 Tonry, J. L., Stubbs, C. W., Lykke, K. R., et al. 2012, ApJ, 750, 99 Trilling, D. E., Mommert, M., Hora, J. L., et al. 2018, AJ, 156, 261 Trilling, D. E., Robinson, T., Roegge, A., et al. 2017, ApJL, 850, L38 
Vincent, M. B., Morse, J. A., Beland, S., et al. 2003, Proc. SPIE, 4841, 367

Vokrouhlický, D., Pravec, P., Durech, J., et al. 2017, A\&A, 598, A91

Williams, G. V. 2017, MPEC, U183

Williams, G. V. 2019a, MPEC, R106

Williams, G. V. 2019b, MPEC, S09

Williams, G. V. 2019c, MPEC, S25
Womack, M., Sarid, G., \& Wierzchos, K. 2017, PASP, 129, 031001

Yang, B., Jewitt, D., \& Bus, S. J. 2009, AJ, 137, 4538

Yang, B., Keane, J., Kelley, M., Protopapa, S., \& Meech, K. J. 2019, CBET, 4672

Ye, Q., Kelley, M. S. P., Bolin, B. T., et al. 2020, AJ, 159, 77

Zhang, Y., \& Lin, D. N. C. 2020, NatAs, in press 